\title{
Dissociation Between the Effects of Damage to Perirhinal Cortex and Area TE
}

\author{
Elizabeth A. Buffalo, ${ }^{2,6}$ Seth J. Ramus, ${ }^{4}$ Robert E. Clark, ${ }^{3}$ Edmond Teng, ${ }^{2}$ \\ Larry R. Squire, ${ }^{1,2,3}$ and Stuart M. Zola ${ }^{1,2,3,5}$ \\ ${ }^{1}$ Veterans Affairs San Diego Healthcare System \\ San Diego, California 92161 USA \\ Departments of ${ }^{2}$ Neurosciences and ${ }^{3}$ Psychiatry \\ University of California, San Diego \\ La Jolla, California 92093 USA \\ ${ }^{4}$ Department of Psychology \\ Boston University \\ Boston, Massachusetts 02134 USA
}

\begin{abstract}
Perirhinal cortex and area TE are immediately adjacent to each other in the temporal lobe and reciprocally interconnected. These areas are thought to lie at the interface between visual perception and visual memory, but it has been unclear what their separate contributions might be. In three experiments, monkeys with bilateral lesions of the perirhinal cortex exhibited a different pattern of impairment than monkeys with bilateral lesions of area TE. In experiment 1, lesions of the perirhinal cortex produced a multimodal deficit in recognition memory (delayed nonmatching to sample), whereas lesions of area TE impaired performance only in the visual modality. In experiment 2 , on a test of visual recognition memory (the visual paired comparison task) lesions of the perirhinal cortex impaired performance at long delays but spared performance at a very short delay. In contrast, lesions of area TE impaired performance even at the short delay. In experiment 3 , lesions of the perirhinal cortex and lesions of area TE produced an opposite pattern of impairment on two visual discrimination tasks, simple object
\end{abstract}

\footnotetext{
${ }^{5}$ Corresponding author.

${ }^{6}$ Present address: Laboratory of Neuropsychology, National Institute of Mental Health, Bethesda, Maryland 20892 USA.
}

discrimination learning (impaired only by perirhinal lesions), and concurrent discrimination learning (impaired only by TE lesions). Taken together, the findings suggest that the perirhinal cortex, like other medial temporal lobe structures, is important for the formation of memory, whereas area TE is important for visual perceptual processing.

\section{Introduction}

It has long been recognized that the anterior inferotemporal cortex, which includes the perirhinal cortex ventromedially and area TE dorsolaterally (Figs. 1 and 2), plays a critical role in visual learning and memory (Gross 1973; Mishkin 1982; Miyashita 1993). The perirhinal cortex has been considered to be part of a memory system in the medial temporal lobe that includes the hippocampal region and the entorhinal, perirhinal, and parahippocampal cortices (for reviews, see Mishkin and Murray 1994; Squire and Zola 1997). Area TE lies immediately adjacent and lateral to the perirhinal cortex and is considered to be the last exclusively visual area in the ventral visual processing stream (Ungerleider and Mishkin 1982). Area TE also originates strong anatomical projections to perirhinal cortex and receives projections from it (Suzuki and Amaral 1994a). With respect to the perirhinal cortex, studies in humans, monkeys, and rats have demonstrated that lesions of perirhinal cortex (or deactivation by cooling) impair performance on tests of learning and memory (Horel et

LEARNING \& MEMORY 6:572-599 @ 1999 by Cold Spring Harbor Laboratory Press ISSN1072-0502/99 \$5.00

$$
\begin{array}{lllllllllllllll}
L & E & A & R & N & I & N & G & \underset{\mathbf{5 7 2}}{\boldsymbol{Z}} & M & E & M & O & R & Y
\end{array}
$$




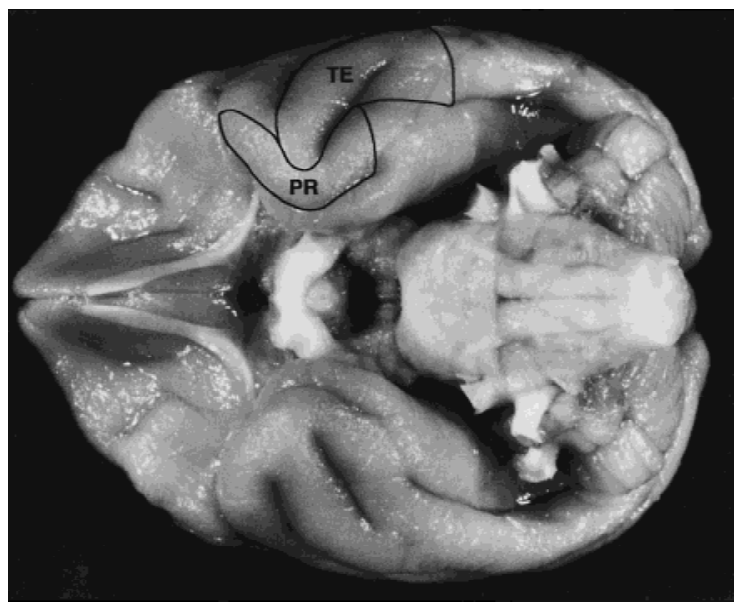

Figure 1: The ventral surface of a macaque monkey brain showing the location of the perirhinal cortex (PR) and inferotemporal cortical area TE (TE). The perirhinal cortex forms a band of cortex along the ventromedial surface of the temporal lobe, lateral to the rhinal sulcus. Area TE is located immediately lateral to the perirhinal cortex and consists of a band of cortex lying primarily on the middle temporal gyrus. See Materials and Methods for details concerning the boundaries of the perirhinal cortex and area TE.

al. 1987; Bachevalier et al. 1993; Mumby and Pinel 1994; Ennaceur et al. 1996; Buckley et al. 1997; Ennaceur and Aggleton 1997; Buffalo et al. 1998a). Lesions of area TE in monkeys (or deactivation by cooling) also impair performance on tests of visual learning and memory (Gross 1973; Dean 1976; Fuster et al. 1981; Mishkin 1982; Buffalo et al. 1998b). It has not been clear precisely how the perirhinal cortex and area TE contribute to performance on visual memory tests or what their different contributions might be.

In three experiments, we compared the performance of monkeys with lesions limited to the perirhinal cortex, monkeys with lesions limited to area TE, and normal monkeys. We explored three ways in which the perirhinal cortex and area TE might be functionally distinct. First, because the perirhinal cortex receives multimodal sensory input whereas area TE receives exclusively visual input, we reasoned that the two areas might differ with respect to their contribution to memory in modalities other than vision. Second, because humans with damage to the perirhinal cortex demonstrate normal visual perception as evidenced by intact visual recognition performance with very short delays (Buffalo et al. 1998a), whereas area TE has been proposed to play a role in visual perceptual processing (Iwai and Mishkin 1968; Gross 1973; Dean 1976; Buffalo et al. 1998b), we reasoned that the two areas might differ in their contributions to visual short-term memory and visual long-term memory. Third, the perirhinal cortex receives direct input from visual areas upstream from area TE (Suzuki and Amaral 1994a), and area TE does not project exclusively to the perirhinal cortex but also originates direct prefrontal and parietal cortical projections as well as subcortical projections to the caudate nucleus (Van Hoesen et al. 1981; Saint-Cyr et al. 1990; Webster et al. 1994). Accordingly, we reasoned that it might be possible to demonstrate a dissociation between the effects of lesions of the perirhinal cortex and area TE on two tasks that involved different kinds of visual discrimination.

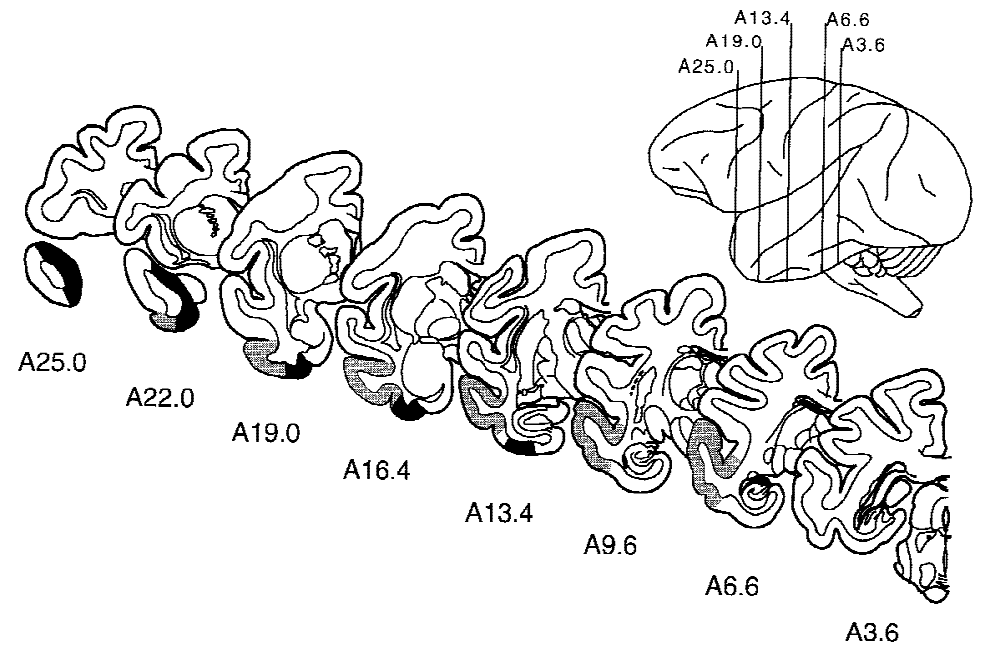

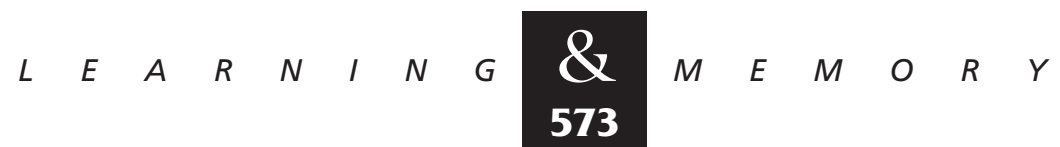

Figure 2: Line drawings of representative coronal sections through the temporal lobe of M. fascicularis adapted from the atlas of Szabo and Cowan (1984). The sections are arranged from rostral (A25.0) to caudal (A3.6), and the rostrocaudal position of each section is indicated in the lateral view. The designations A25.0, A22.0, and so on, specify distances anterior (A) to the intra-aural line. The boundaries of the perirhinal cortex are indicated in black, and the boundaries of area TE are indicated in gray. 
Buffalo et al.

Table 1: Behavioral performance

\begin{tabular}{|c|c|c|c|c|c|c|c|c|c|c|c|c|c|}
\hline \multirow[b]{5}{*}{ Group } & & \multicolumn{12}{|c|}{ Experiment 1} \\
\hline & & \multicolumn{7}{|c|}{ V-DNMS } & \multicolumn{5}{|c|}{ T-DNMS } \\
\hline & & \multirow{2}{*}{\multicolumn{2}{|c|}{$\begin{array}{l}\text { trials to } \\
\text { criterion }\end{array}$}} & \multicolumn{5}{|c|}{ delays } & \multirow[b]{3}{*}{ trials } & \multicolumn{4}{|c|}{ delays } \\
\hline & & & & 8 & 15 & 1 & 10 & 40 & & 8 & 15 & 1 & 10 \\
\hline & & 1 & II & sec & $\mathrm{sec}$ & $\min$ & $\min$ & $\min$ & & sec & sec & $\min$ & $\min$ \\
\hline \multirow[t]{19}{*}{ Normal } & $1^{\mathrm{a}}$ & 180 & 0 & 92 & 93 & 88 & 73 & 75 & - & - & - & - & - \\
\hline & $2^{\mathrm{a}}$ & 440 & 0 & 92 & 94 & 94 & 70 & 69 & - & - & - & - & - \\
\hline & $3^{a}$ & 220 & 0 & 91 & 93 & 88 & 79 & 72 & - & - & - & - & - \\
\hline & $4^{\mathrm{a}}$ & 120 & 20 & 92 & 97 & 91 & 83 & 76 & - & - & - & - & - \\
\hline & $5^{\mathrm{a}}$ & 415 & 0 & 91 & 93 & 88 & 71 & 64 & - & - & - & - & - \\
\hline & 6 & 189 & 0 & 94 & 99 & 96 & 86 & 74 & 359 & 91 & 88 & 92 & 68 \\
\hline & 7 & 180 & 0 & 95 & 95 & 91 & 68 & 76 & 292 & 90 & 94 & 86 & 66 \\
\hline & 8 & 0 & 20 & 92 & 93 & 91 & 85 & 78 & 522 & 91 & 93 & 85 & 80 \\
\hline & 9 & 0 & 20 & 92 & 93 & 92 & 85 & 78 & 280 & 91 & 92 & 88 & 84 \\
\hline & 10 & 220 & 60 & 93 & 94 & 90 & 73 & 67 & 446 & 91 & 92 & 84 & 76 \\
\hline & $11^{\mathrm{b}}$ & - & - & - & - & - & - & - & - & - & - & - & - \\
\hline & $12^{\mathrm{b}}$ & - & - & - & - & - & - & - & - & - & - & - & - \\
\hline & $13^{b}$ & - & - & - & - & - & - & - & - & - & - & - & - \\
\hline & $14^{\mathrm{b}}$ & - & - & - & - & - & - & - & - & - & - & - & - \\
\hline & $15^{\mathrm{b}}$ & - & - & - & - & - & - & - & - & - & - & - & - \\
\hline & $16^{\mathrm{b}}$ & - & - & - & - & - & - & - & - & - & - & - & - \\
\hline & $17^{\mathrm{b}}$ & - & - & - & - & - & - & - & - & - & - & - & - \\
\hline & $18^{\mathrm{b}}$ & - & - & - & - & - & - & - & - & - & - & - & - \\
\hline & Mean & 196 & 12 & 92 & 94 & 91 & 77 & 73 & 380 & 91 & 92 & 85 & 75 \\
\hline \multirow[t]{6}{*}{ PR } & 1 & 860 & 480 & 90 & 84 & 88 & 72 & 70 & 704 & 90 & 77 & 63 & 48 \\
\hline & 2 & 100 & 0 & 92 & 79 & 83 & 66 & 72 & - & - & - & - & - \\
\hline & 3 & 100 & 460 & 90 & 92 & 85 & 69 & 71 & 464 & 90 & 83 & 68 & 42 \\
\hline & 4 & 0 & 200 & 91 & 83 & 76 & 67 & 51 & 573 & 90 & 75 & 78 & 52 \\
\hline & 5 & 2034 & 0 & 91 & 87 & 85 & 71 & 66 & 501 & 90 & 74 & 65 & 46 \\
\hline & Mean & 619 & 228 & 91 & 85 & 83 & 69 & 66 & 561 & 90 & 77 & 69 & 47 \\
\hline \multirow[t]{6}{*}{ TE } & 1 & 440 & 180 & 91 & 88 & 82 & 74 & 60 & - & - & - & - & - \\
\hline & 2 & 740 & 0 & 92 & 84 & 76 & 76 & 67 & - & - & - & - & - \\
\hline & 3 & 328 & 0 & 93 & 90 & 88 & 65 & 55 & 288 & 90 & 87 & 83 & 70 \\
\hline & 4 & 1077 & 0 & 91 & 91 & 93 & 66 & 66 & - & - & - & - & - \\
\hline & 5 & 760 & 40 & 90 & 93 & 90 & 66 & 66 & 80 & 90 & 90 & 83 & 70 \\
\hline & Mean & 669 & 44 & 91 & 89 & 86 & 69 & 63 & 123 & 90 & 89 & 83 & 70 \\
\hline
\end{tabular}

\section{Materials and Methods}

\section{SUBJECTS}

Twenty-eight male cynomolgus monkeys ( $\mathrm{Ma}$ caca fascicularis) were tested, all weighing between $3.1 \mathrm{~kg}$ and $5.2 \mathrm{~kg}$ at the start of the experiment. Five monkeys received bilateral lesions of the perirhinal cortex (PR group). Five monkeys re- ceived bilateral lesions of inferotemporal cortical area TE (TE group). The remaining eighteen monkeys comprised unoperated control groups used in the individual experiments ( $\mathrm{N}$ group; see Table 1 ).

SURGERY

Monkeys were anesthetized using Isofluorane gas and placed in a stereotaxic headholder that

$$
\begin{array}{lllllllllllllll}
L & E & A & R & N & I & N & G & \mathbf{8} & M & E & M & O & R & Y \\
\mathbf{5 7 4} & & & & & &
\end{array}
$$


Table 1: (Continued)

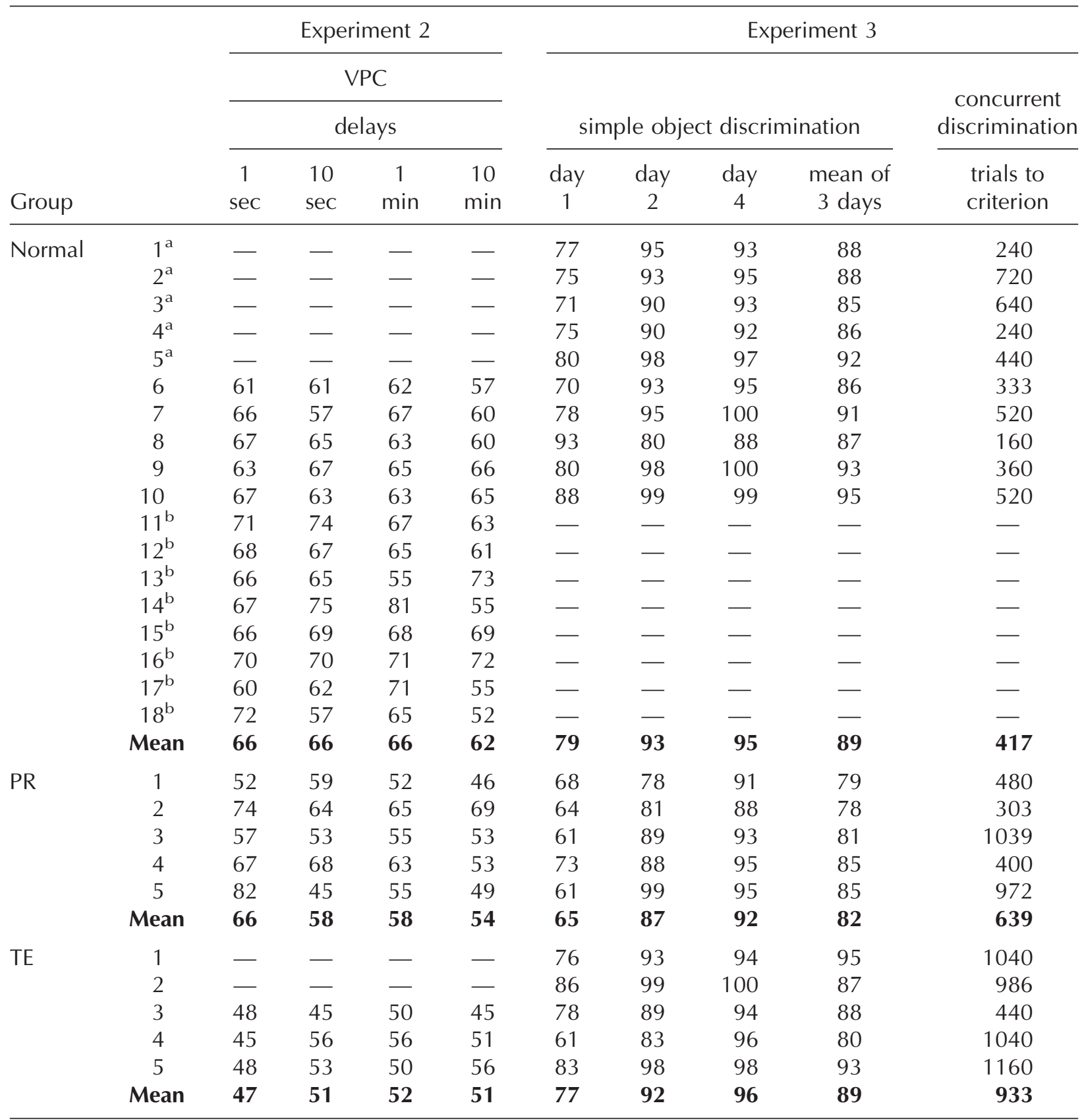

(V-DNMS) Visual delayed nonmatching to sample; (T-DNMS) tactual delayed nonmatching to sample; (VPC) visual paired-comparison task.

${ }^{a}$ Monkeys N1-N5 were not available for testing on the T-DNMS or VPC tasks.

${ }^{\mathrm{b}}$ Monkeys N11-N18 were tested only on the VPC task.

allowed unobstructed access to the temporal portion of the skull. The temporal muscles were exposed and fully retracted, and the zygomatic arches were removed. Bilateral openings were made in the skull to expose the anterior and ven- trolateral portions of the temporal lobe, and the dura was opened. The pial surface over the intended lesions was first cauterized, and the cauterized tissue together with the underlying cortical tissue was then removed by suction using a glass

$$
\begin{array}{lllllllllllllll}
\hline & E & A & R & N & I & N & G & \boldsymbol{Q} \\
\mathbf{5 7 5} & M & E & M & O & R & Y
\end{array}
$$




\section{Buffalo et al.}

pipette with an angled tip. Cortical tissue was removed slowly until the white matter was visualized. The dura was then sewn, and the wound was closed in anatomical layers.

\section{LOCATION OF THE PERIRHINAL} CORTEX AND AREA TE

Before describing the intended lesions of each of the animals in the two operated groups, it is useful to summarize briefly the location and boundaries of the perirhinal cortex and area TE. Our description of the boundaries of the perirhinal cortex follow those of Suzuki and Amaral (1994a). The perirhinal cortex forms a band of cortex along the ventromedial surface of the temporal lobe (Figs. 1 and 2). It consists of a smaller, medially situated area 35 and a larger, laterally situated area 36 (Brodmann 1909). The perirhinal cortex is located approximately lateral to the rhinal sulcus and follows the rhinal sulcus from its anterior border at the frontotemporal junction (limen insula) through its caudal extent on the ventral surface of the brain. The perirhinal cortex typically extends a few millimeters beyond the caudal limit of the rhinal sulcus. At its most rostral and dorsal extent, the perirhinal cortex makes up the medial portion of what is typically referred to as the temporal pole or area TG. Throughout most of its rostrocaudal extent, the perirhinal cortex is bordered laterally by area TE. At its most caudal end, the perirhinal cortex is bordered laterally by the rostral portion of the parahippocampal cortex. The structures that form the medial border of the perirhinal cortex vary at different rostrocaudal levels. At rostral levels, the medial border is formed by the piriform and periamygdaloid cortices. At more caudal levels, the entorhinal cortex forms the medial border of the perirhinal cortex.

Area TE is located immediately lateral to the perirhinal cortex at most levels and consists of a band of cortex lying primarily on the middle temporal gyrus (Figs. 1 and 2). Area TE corresponds roughly to Brodmann area 21 (dorsal) and the lateral part of area 20 (ventral) (Brodmann 1909). At its rostral extent, the perirhinal cortex forms the medial border of area TE. At its caudal end, the medial border is formed by the parahippocampal cortex. Throughout its rostrocaudal extent, area TE is bordered laterally by superior temporal cortex. Area TEO forms the posterior border of area TE. The description of the area TE-perirhinal cortex border has varied across studies. Based on recent anatomical studies (Suzuki and Amaral 1990, $1994 a$, b) in $M$. fascicularis, we have placed the area TE-perirhinal cortex border on the medial bank of the AMTS, $\sim 2 \mathrm{~mm}$ more lateral than in previous studies (Meunier et al. 1993; Eacott et al. 1994).

\section{INTENDED PR AND TE LESIONS}

For the perirhinal lesions, $\sim 3-4 \mathrm{~mm}$ of cortex lateral to the full rostrocaudal extent of the rhinal sulcus was removed (see Figs. 1 and 2). The medial portion of the temporal polar region was also removed. This lesion was intended to include the two distinct cytoarchitectonic regions that compose the perirhinal cortex, that is, areas 35 and 36 of Brodmann (Brodmann 1909).

For the lesions of area TE, the middle temporal gyrus and a portion of the posterior inferior temporal gyrus were removed (see Figs. 1 and 2). The intended lesion was bounded laterally by the ventral bank of the STS. We intentionally did not include the ventral bank of the STS in the lesion to avoid damaging the large vessel that runs in this sulcus. The tissue in the ventral bank of the STS constitutes $\sim 35 \%$ of area TE. Accordingly, the intended lesions in the present study included, at most, $\sim 65 \%$ of area TE. At its rostral and medial extent, the intended lesion was bounded by the fundus of the AMTS through the rostral half of this sulcus. At its caudal extent, the medial border of the intended lesion extended to the occipito-temporal sulcus. At its rostral extent, this was a slightly conservative boundary (the border between area TE and the perirhinal cortex typically lies on the medial lip of the AMTS), to avoid any damage to the perirhinal cortex. At its rostral border, the intended lesion extended to an imaginary line connecting the tips of the STS and the AMTS. At its caudal border, the lesion extended to $\sim 10 \mathrm{~mm}$ in front of the ascending inferior occipital sulcus.

\section{HISTOLOGICAL VERIFICATION OF LESIONS}

After completion of behavioral testing, operated monkeys were administered an overdose of sodium pentobarbital. Following deep anesthetization measured by loss of the corneal reflex, the animals were sacrificed by transcardial perfusion of fixatives. They were perfused with a solution of $0.9 \%$ saline followed by $10 \%$ buffered formalin. The brains were cryoprotected in glycerol solutions

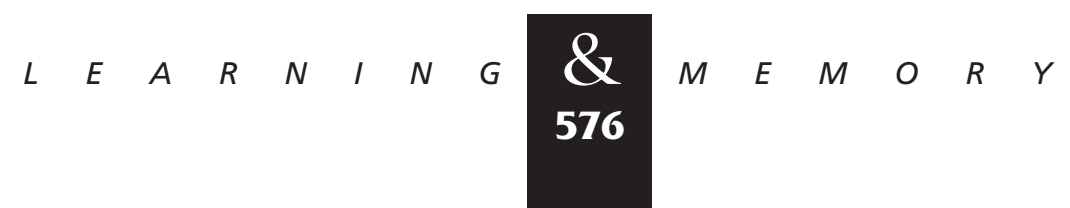


and sectioned in the coronal plane at $50 \mu \mathrm{m}$ on a freezing microtome. Every fifth section was mounted and stained with thionin to assess the extent of the lesions.

The brains from four monkeys that matched the average weight of each of the two operated groups were used as a histological control group. Thionin-stained sections from the four monkeys in the histological control group, extending from the tips of the temporal poles to the caudal extent of area TE, were scanned into a Macintosh G3 computer at $0.50-\mathrm{mm}$ intervals. Cortical areas were classified on the basis of cytoarchitectonic characteristics using a light microscope (Leica WILD 3Z) and were marked on the computerized images of each section. The cytoarchitectonic criteria used to determine the perirhinal cortex-area TE border follow those of Suzuki and Amaral (pers. comm.). Briefly, distinctions between the lateral portion of the perirhinal cortex and area TE include the following: layer II of the perirhinal cortex contains distinct aggregates of cells, whereas area TE does not; layer III of area TE is more radially oriented than in the perirhinal cortex; layer IV in area TE is thicker and more densely populated than in the perirhinal cortex; and area TE, unlike the perirhinal cortex, is characterized by a clear separation between layers V and VI. Because there are no clear cytoarchitectonic criteria by which to distinguish area TE from area TEO, we designated the caudal extent of area TE as the beginning of the posterior middle-temporal sulcus. Using NIH Image software, areal measurements were made of area TE and the perirhinal cortex in each monkey.

The area of each of the two cortical regions was measured by calculating, at each rostrocaudal level, the medial-lateral extent of cortical layer I of area TE (including the ventral bank of the superior temporal sulcus) and the perirhinal cortex. This measurement was then multiplied by the interval between the sections $(0.50 \mathrm{~mm})$, and the sum of these products then provided an areal estimate for each of the two cortical regions. This same procedure was followed for both the left and right temporal lobes, and the estimates for each hemisphere were averaged. The estimates from all four control monkeys were averaged, and this value was used as the estimate of the normal area for the two cortical regions.

Thionin-stained sections at $0.50-\mathrm{mm}$ intervals were scanned and measured for each monkey in the two surgical groups in the same way as for the four histological control monkeys. The final calcu- lation yielded an estimate of spared cortex in the lesioned monkeys. This value was then divided by the estimate of the averaged normal area for the same cortical region, thereby yielding an estimate (from 0 to 1 ) of the proportion of spared tissue. This estimate was then subtracted from 1 and multiplied by 100 to obtain an estimate of the percentage of bilaterally damaged tissue. For example, animal PR 2 was estimated to have $15.15 \mathrm{~mm}^{2}$ of bilaterally spared perirhinal cortex. This value was divided by the mean area of perirhinal cortex calculated from the four histological control animals $\left(112.80 \mathrm{~mm}^{2}\right)$, to yield 0.13 as the proportion of bilaterally spared tissue and $100(1-0.13)=87$ as the percentage of perirhinal cortex damaged. Because of the large variation between monkeys in the surface areas within the temporal lobe, the amounts of cortical damage in the animals with lesions are necessarily only estimates.

\section{BEHAVIORAL TASKS}

Findings from six behavioral tasks will be presented. The actual order in which the tasks were given is as follows: (1) visual delayed nonmatching to sample (V-DNMS), (2) simple object discrimination, (3) concurrent discrimination learning, (4) retesting of V-DNMS, (5) visual paired-comparison (VPC), and (6) tactual delayed nonmatching to sample (T-DNMS). The findings from the six tasks have been grouped to form three experiments. Experiment 1 addressed whether lesions of PR or TE produced a multimodal memory impairment and included the V-DNMS and T-DNMS tasks. Experiment 2 determined the effects of PR and TE lesions on both short-term and long-term visual recognition memory using the VPC task. Experiment 3 determined the effects of PR and TE lesions on visual discrimination learning and included the simple object discrimination and concurrent discrimination tasks.

The three experiments necessarily have different groups of control animals (see Table 1). Behavioral tasks 1-4 (see above) form our standardized test battery by which we have routinely assessed the effects of lesions. All 10 unoperated control monkeys (N1-N10) that have been tested on this same battery were used as the control group for the V-DNMS task and the retest of the V-DNMS task in experiment 1 and the two discrimination tasks in experiment 3. After completion of the standardized battery, not all of the original 10 control monkeys could be given the other tests. Monkeys N6-

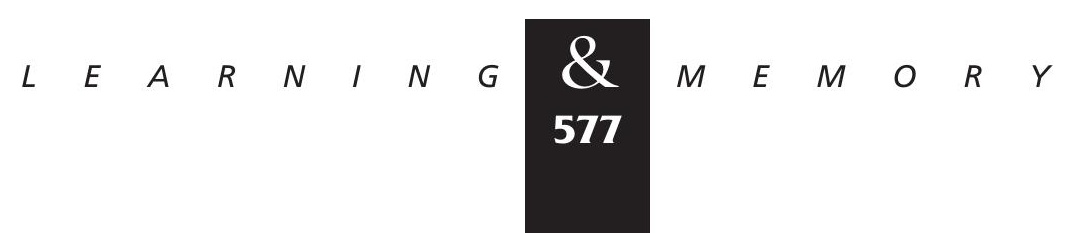


N10 were tested on the T-DNMS (experiment 1). A larger group of 13 control monkeys (N6-N18) were tested on the VPC task. This group includes all unoperated monkeys tested on this task in our laboratory to date. Only five of these control monkeys for the VPC task (N6-N10) had exactly the same behavioral history as the operated groups. However, the difference in behavioral history did not affect performance (N6-N10 vs. N11-N18: no differences in performance at each delay, all Ps $>0.10)$.

All groups of animals were given some additional tasks during the course of behavioral testing. Specifically, after the retest of the V-DNMS task, all operated monkeys were tested on several other tasks including a motor skill task as well as spatial and nonspatial memory tasks. It is important to note that both operated groups had exactly the same testing history.

\section{Experiment 1: Do the Perirbinal Cortex and Area TE Contribute to Memory in More Than One Modality?}

\section{Materials and Methods}

\section{SUBJECTS}

The five PR monkeys, the five TE monkeys, and control monkeys N1-N10 were tested on the V-DNMS task. The 10 control monkeys included all of the unoperated monkeys with the same behavioral history tested in our laboratory to date. For the T-DNMS task, control monkeys N1-N5 were tested together with four of the PR monkeys and two of the TE monkeys. Monkeys N6-N10 were not available for testing on the T-DNMS task. Additionally, prior to administration of the T-DNMS task, one monkey from the PR group (PR 2) had to be euthanized as a result of a tumor. Two monkeys from the TE group (TE 1 and TE 2) had to be euthanized as a result of gastrointestinal disorder, and one monkey from the TE group (TE 4) developed behavioral problems that prevented his participation.

\section{BEHAVIORAL TESTING}

All testing was conducted in the Wisconsin General Testing Apparatus (WGTA; Harlow and Bromer 1938). In this apparatus, the experimenter and monkey face opposite sides of a testing tray that has three food wells. A sliding opaque door separates the monkey from the testing tray, and a sliding door with a one-way mirror separates the experimenter from the tray. The one-way mirror allows the experimenter to observe the monkey but prevents the monkey from seeing the experimenter. During a trial, the experimenter lowers the one-way mirror and raises the opaque door, allowing the monkey to respond to the objects on the testing tray.

Each operated group was allowed 6-8 weeks of recovery prior to the start of behavioral testing. During four to six daily sessions of pretraining, operated monkeys and control monkeys learned to obtain food by displacing objects that covered any of three food wells located on a stimulus tray in front of the testing chamber.

\section{V-DNMS}

In this task, monkeys first displaced a single object (the sample object) covering the center well of the three-well food tray to obtain a food reward. The opaque door was then lowered between the monkey and the wells. After an 8-sec delay, the door was raised to expose two objects, the previously presented sample object and a novel object, each covering one of the two lateral food wells. Monkeys had to displace the novel object to obtain a raisin reward. The position of the novel object (covering the right or left food well) varied pseudorandomly on each trial (Gellerman 1933). Twenty trials per day were given, with an intertrial interval of $20 \mathrm{sec}$. Each trial used a new pair of objects selected randomly from a collection of $>400$ junk objects. After reaching a learning criterion of $90 \%$ correct ( 90 correct choices in 100 consecutive trials), monkeys were tested with successively longer delays of $15 \mathrm{sec}, 1 \mathrm{~min}, 10 \mathrm{~min}$, and 40 min between the sample and choice trials. Monkeys were tested for 100 trials with the 15-sec and 1-min delays (20 trials per day for 5 days), and 50 trials with the 10-min and 40-min delays (5 trials per day for 10 days).

Seven to twelve months after the first administration of the V-DNMS task, monkeys were retested on the V-DNMS task. The task was given in the identical way it had been given originally.

\section{T-DNMS}

Four PR monkeys, 2 TE monkeys, and 5 control monkeys were tested on the T-DNMS task. The

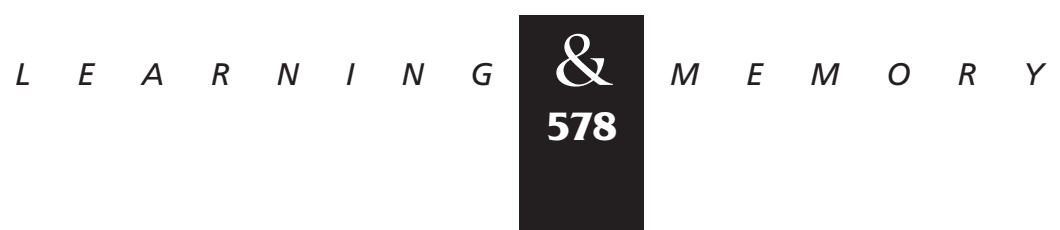


procedure for the T-DNMS task was modified from that reported by Suzuki et al. (1993). An opaque barrier ( $72 \mathrm{~cm}$ long by $18 \mathrm{~cm}$ high) was inserted between the testing cage and the stimulus tray to block the monkey's view of the objects. A rectangular platform ( $8 \mathrm{~cm}$ wide by $45.5 \mathrm{~cm}$ long by 7.5 $\mathrm{cm}$ high), with its long axis oriented parallel to the opaque barrier, was secured to the stimulus tray of the WGTA $\sim 1.5 \mathrm{~cm}$ in front of the opaque barrier on the experimenter's side of the apparatus. The platform contained two round wells, $6.4 \mathrm{~cm}$ in diameter and spaced $8 \mathrm{~cm}$ apart, measured from the center of each well. The junk objects used in the tactual task were mounted on round disks that fit snugly into the wells. The experimenter could manually lock the objects mounted on the round disks into place by moving a lever. A pool of $\sim 150$ objects differing in shape, size, and texture was used for the tactual task. At the bottom of each of the wells that held the round disks was a smaller well $(1.5 \mathrm{~cm}$ diam.) that held the food rewards. The opaque barrier, the platform, and the tactual objects were constructed so that monkeys could easily grasp objects that were presented on the other side of the opaque barrier but could not see the objects from any position in the testing box. Behavioral observation during pretraining and administration of the T-DNMS task confirmed that the monkeys could not see the objects from any position in the testing box. For example, during testing, the monkeys did not attempt to peer over the opaque barrier before making a choice but simply reached over the barrier to grasp the objects. Moreover, even when the monkeys were performing at criterion level on the T-DNMS task, they were equally likely to grasp the correct or incorrect object during their first contact with the objects.

\section{PRETRAINING}

Pretraining for the T-DNMS task was administered in four phases. During the first three phases of pretraining, a single object was used and was always rewarded.

In phase 1 , the platform but not the opaque barrier was used, and monkeys had full view of the training object. The animals learned to displace the training object and retrieve a food reward when the object was placed randomly in one of the two object wells. Initially, the object was not locked into the object well and could easily be removed. When the animals readily displaced the unlocked object from the well, the object was then locked into place, and the animals were required to tug on the object three times before it was released by the experimenter. Pilot experiments showed that if monkeys were allowed to displace unlocked objects, they would quickly knock the objects out of the wells while searching for the reward without examining the objects tactually. Thus, the procedure of requiring the animals to tug on the object a fixed number of times helped ensure that all animals received equal tactual exposure to the objects. This same procedure was also used during the regular administration of the T-DNMS task. During this phase of pretraining, two tones lasting $\sim 1$ sec each, generated from a minielectronic synthesizer, were presented immediately after the rewarded object was displaced to alert the animal to the presence of a reward. The tones were used throughout tactual pretraining and throughout the T-DNMS task. Phase 1 required one or two testing sessions.

In phase 2, a transparent barrier was inserted into the WGTA between the testing cage and the stimulus tray. The animals could still see the object and learned to reach over the transparent barrier to displace the object. Again, they were required to tug three times before the object was released, and the tones were presented each time the object was successfully displaced. This phase required between two and four testing sessions.

In phase 3, the animal's view of the training object was gradually decreased by attaching opaque panels that progressively covered more of the transparent barrier. At various times during this phase, the monkeys stopped performing the task, and their view of the objects had to be increased to encourage them to work again. Gradually, the animals learned to displace the object and retrieve the food reward while reaching over a barrier that completely blocked visual access. This phase required 5-10 training sessions.

Pilot experiments showed that monkeys initially had some difficulty displacing flat objects. This difficulty affected the monkey's choice and appeared to interfere with learning of the task. Therefore, phase 4 of pretraining was initiated to overcome the initial difficulty observed in displacing certain objects. In this phase, the monkeys were given practice displacing 10 training objects with the opaque panels in place. These 10 training objects varied in size, shape, and texture and provided the monkeys with practice in displacing objects that varied with respect to the difficulty with which they could be displaced. These 10 objects

$$
\begin{array}{lllllllllllllll}
\mathbf{Q} & E & A & R & N & I & N & G & M & E & M & O & R & Y \\
\mathbf{5 7 9} & & & & & &
\end{array}
$$




\section{Buffalo et al.}

were not used subsequently on the T-DNMS task, but were used only in pretraining. Monkeys were presented with one object at a time, and each object was rewarded. In a given session, monkeys were presented with each of the 10 objects twice in random order. Training in this phase was continued until the monkey quickly and easily displaced all 10 objects twice within a given session. This phase required two to four training sessions.

TRAINING

Monkeys first reached over the opaque barrier and tugged three times on a sample object locked into one of the wells. After tugging three times on the object, the monkey was presented with a single tone that signaled the end of the sample phase. The monkey was not allowed to displace the sample object, nor was the monkey rewarded during the sample phase. Following the presentation of the tone, an opaque door was then lowered to block the monkey's access to the platform. After an 8-sec delay, the opaque door was raised, and monkeys could reach over and palpate the sample object and a novel object that covered the two object wells. The monkeys were required to displace the novel object to receive the food reward. Only displacement of the correct object was followed immediately by two tones. Once an object was displaced, no correction was allowed. An intertrial interval of $20 \mathrm{sec}$ was used. The sample object and the correct choice object appeared over the left or right object well in a modified Gellerman (1933) sequence. Each daily session consisted of 20 trials and was comprised of five trials with the sample and correct choice objects both on the left, five trials with the sample and correct choice objects both on the right, five trials with the sample object on the left and the correct choice object on the right, and five trials with the sample object on the right and the correct choice object on the left. These four trial types were mixed throughout the testing session. Each trial of a given session used a new pair of tactual objects selected from a collection of approximately 150 junk objects. After reaching a learning criterion of $90 \%$ correct $(90$ correct choices in 100 consecutive trials), monkeys were tested with successively longer delays of $15 \mathrm{sec}, 1 \mathrm{~min}$, and $10 \mathrm{~min}$ between the sample and choice trials. Monkeys were tested for 100 trials with the 15-sec and 1-min delays (20 trials per day for 5 days) and 50 trials with the 10-min delay ( 5 trials per day for 10 days).

\section{Results}

HISTOLOGICAL RESULTS: DESCRIPTIONS OF THE LESIONS IN THE PR GROUP

GENERAL OBSERVATIONS

For each of the lesions in the PR group, the extent of removal will be described at four rostrocaudal levels: at the level of the temporal pole, corresponding roughly to A25.0 in Figure 2; at the level of the region rostral to the amygdala, corresponding to A19.0 in Figure 2; at the level of the amygdala, corresponding to A16.4 in Figure 2; and at the level of the rostral hippocampus, corresponding to A13.4 in Figure 2. On average, the PR group sustained an estimated $74 \%$ damage to the perirhinal cortex (PR 1 =73\%, PR 2=87\%, PR $3=65 \%$, PR $4=69 \%$, PR 5=77\%). All monkeys sustained some damage to the perirhinal cortex at the polar region, although in most cases the most dorsal part of area 36 was spared at the pole. In all cases, there was extensive damage to the perirhinal cortex beginning rostral to the amygdala and extending through the caudal extent of the perirhinal cortex. The amount of unintended damage outside the perirhinal cortex was minimal in all but one case (PR 1). Monkeys PR 2-PR 5 sustained little or no direct damage to area TE. Monkey PR 1 sustained extensive unilateral damage to area TE (see details below). Monkeys PR 2-PR 5 sustained minimal damage to rostral parahippocampal cortex, whereas monkey PR 1 sustained moderate unilateral damage to the parahippocampal cortex. The entorhinal cortex was completely spared in all cases. Additionally, in none of the cases was there damage to the amygdala or the hippocampal region. There was minimal white matter damage in all cases.

The extent of the lesion in each monkey of the PR group is plotted on representative coronal sections (Fig. 3). The rostrocaudal levels used to illustrate the lesions are the same as those used in Figure 2. The full rostrocaudal extent of the lesion in animal PR 2 is shown in Figure 4. The lesion in this monkey most closely approximated the intended lesion and will be described first. Then, shorter, comparative descriptions of the lesions in the other animals in the PR group are provided.

PR 2

At its rostral extent, the lesion in monkey PR 2 began at the level of the temporal pole with dam-

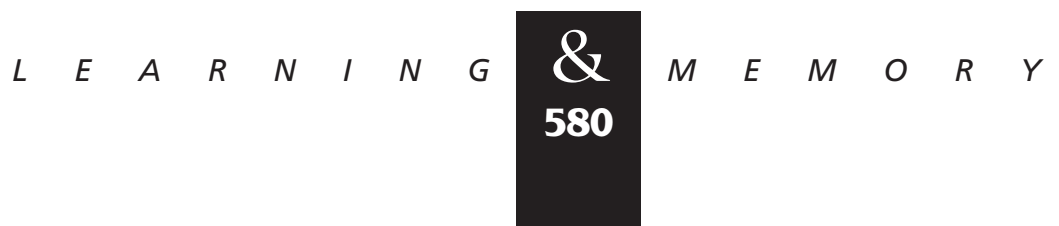




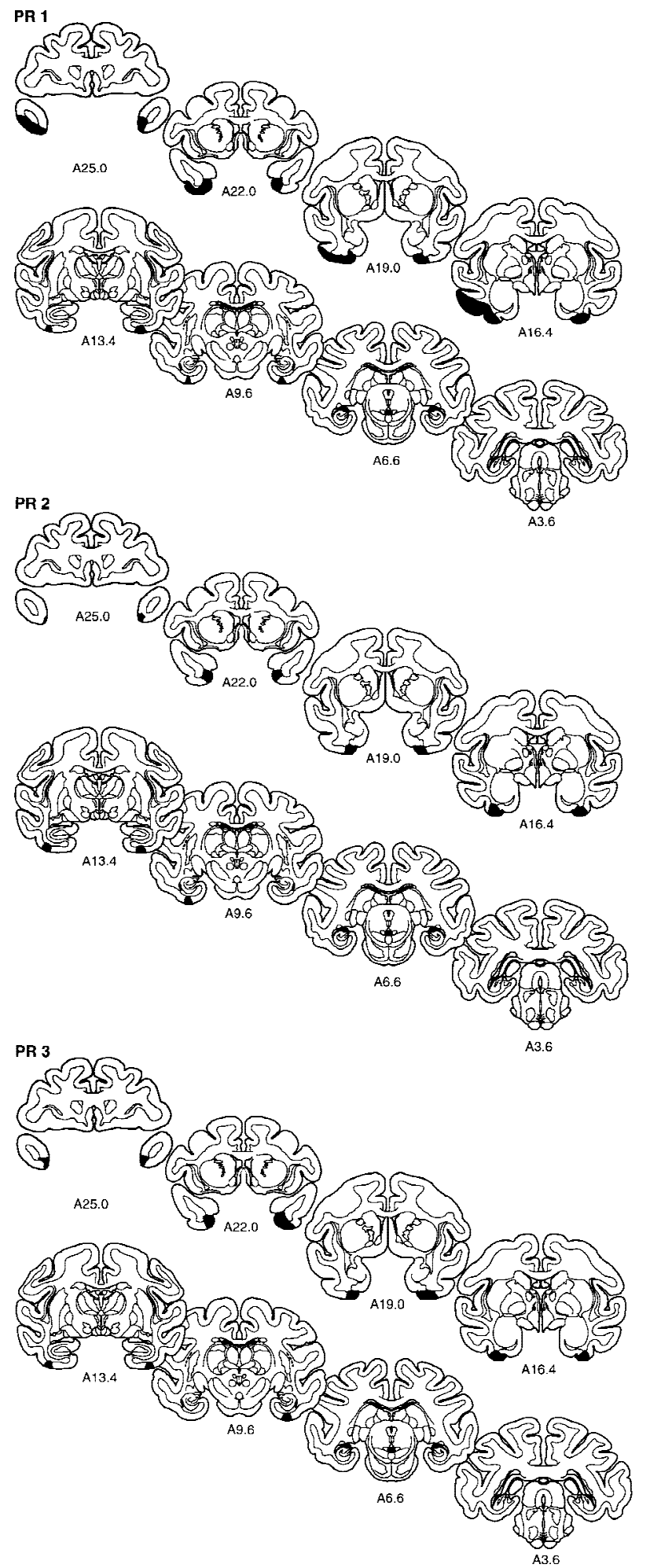

age involving cortical layers I and II and encompassing about the ventral one-third of the medial portion of the pole on the left side (A25.0; Fig. 4A). On the right side, the ventromedial portion of the

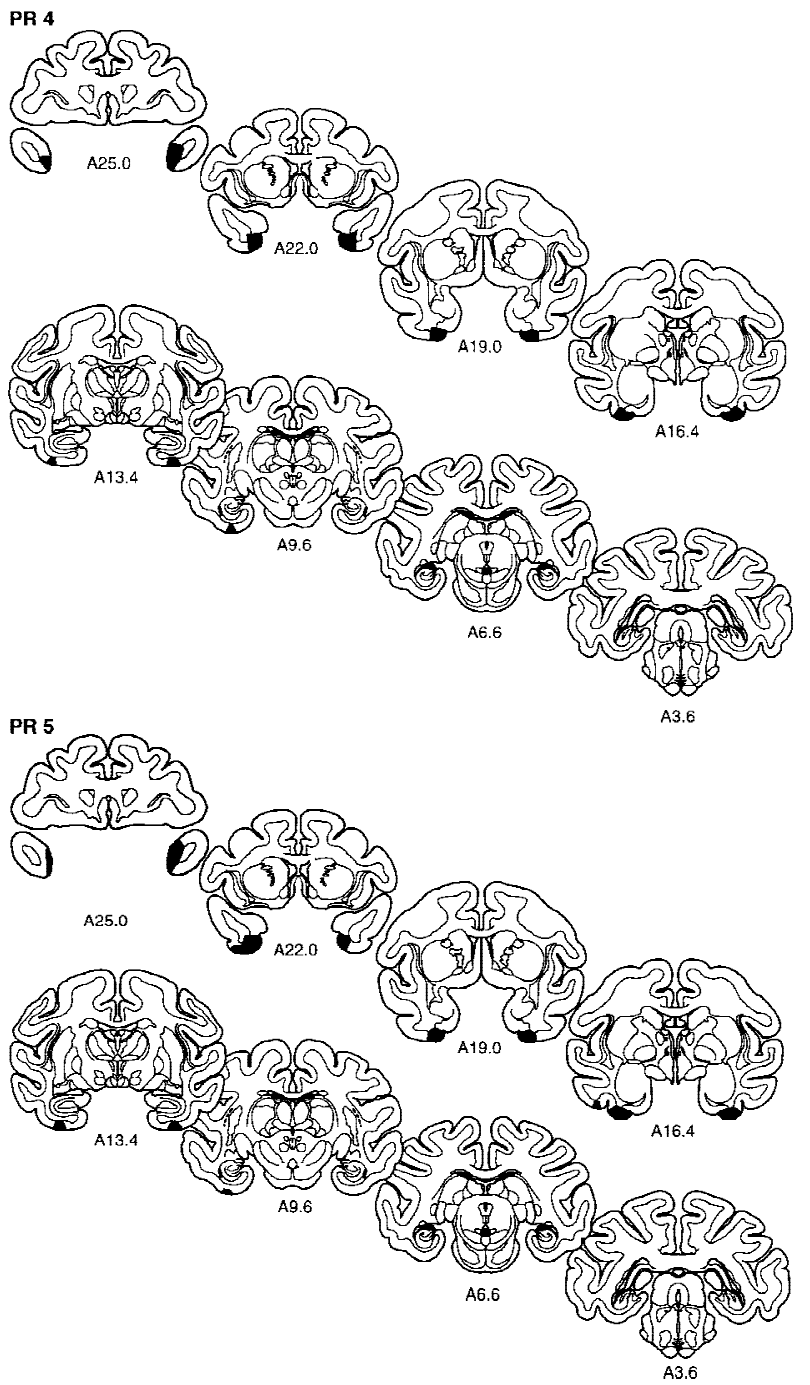

Figure 3: The extent of the lesions of perirhinal cortex in each of the five monkeys in the PR group (PR 1-PR 5) is plotted on representative coronal sections redrawn from the atlas of Szabo and Cowan (1984). In each case, the area of the lesion is indicated in black. The rostrocaudal level is indicated below each section (see Figs. 1 and 2 for borders of the perirhinal cortex).

temporal pole was more extensively damaged and included approximately one-half of the medial portion of the temporal pole. Rostral to the amygdala (A22.0; Fig. 4B), the lesion included, bilaterally, all

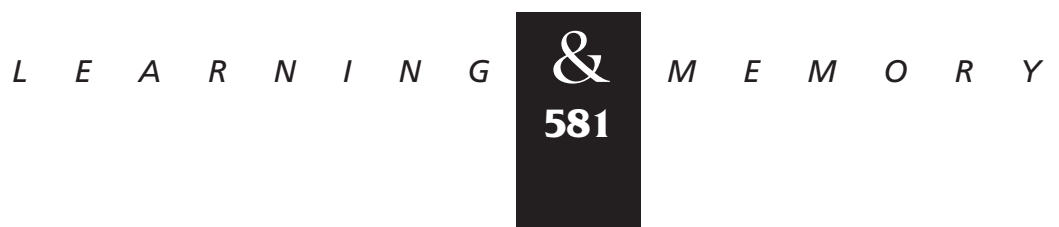




\section{Buffalo et al.}

layers of area 36. At this level, much of area 35 was spared along with the dorsal part of area 36. At a more caudal level, at the level of the amygdala (A16.4; Fig. 4D), there was nearly complete removal of the perirhinal cortex bilaterally, including both areas 35 and 36 . The removal of the perirhinal cortex continued through the caudal extent of the perirhinal cortex, at the level of the rostral hippocampus (A13.4; Fig. 4F), and included minimal damage to the parahippocampal cortex, which extended in the rostrocaudal plane $\sim 2 \mathrm{~mm}$ on the left side and $\sim 1 \mathrm{~mm}$ on the right side. The lesion in
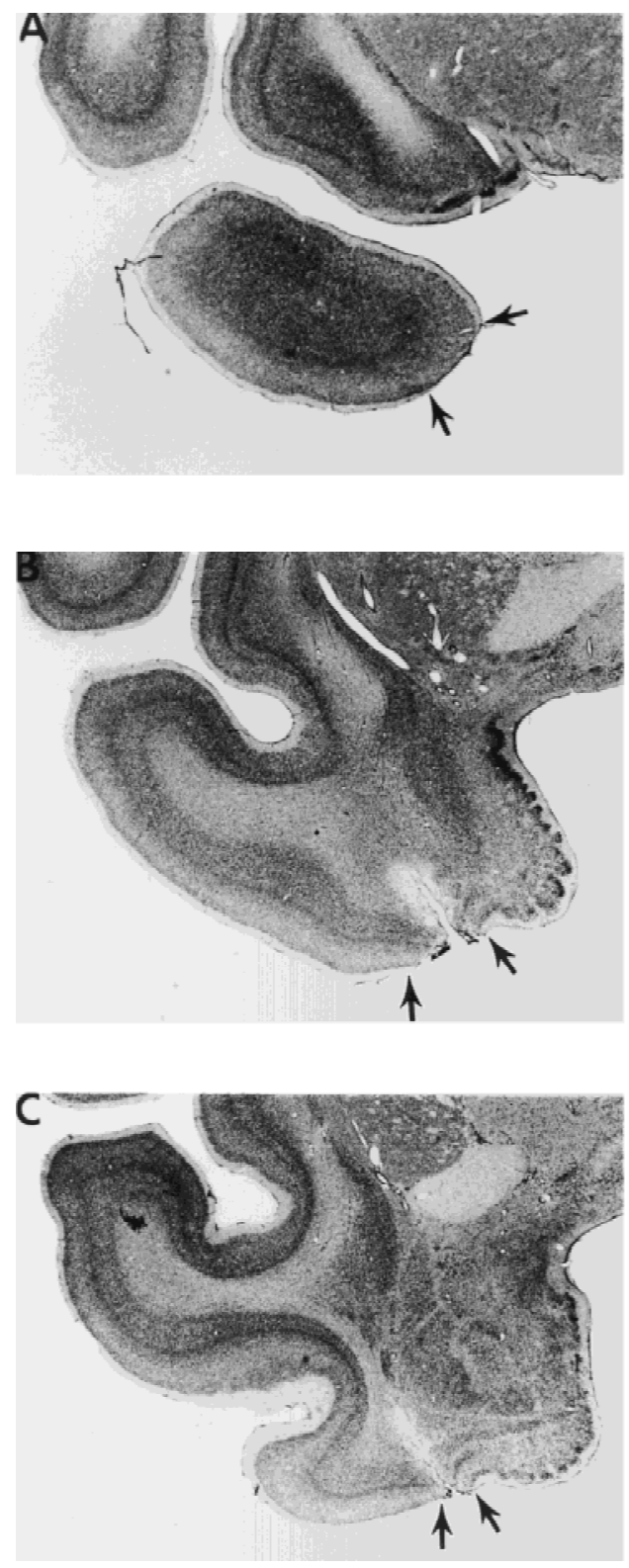

Figure 4: (Continued on facing page.)
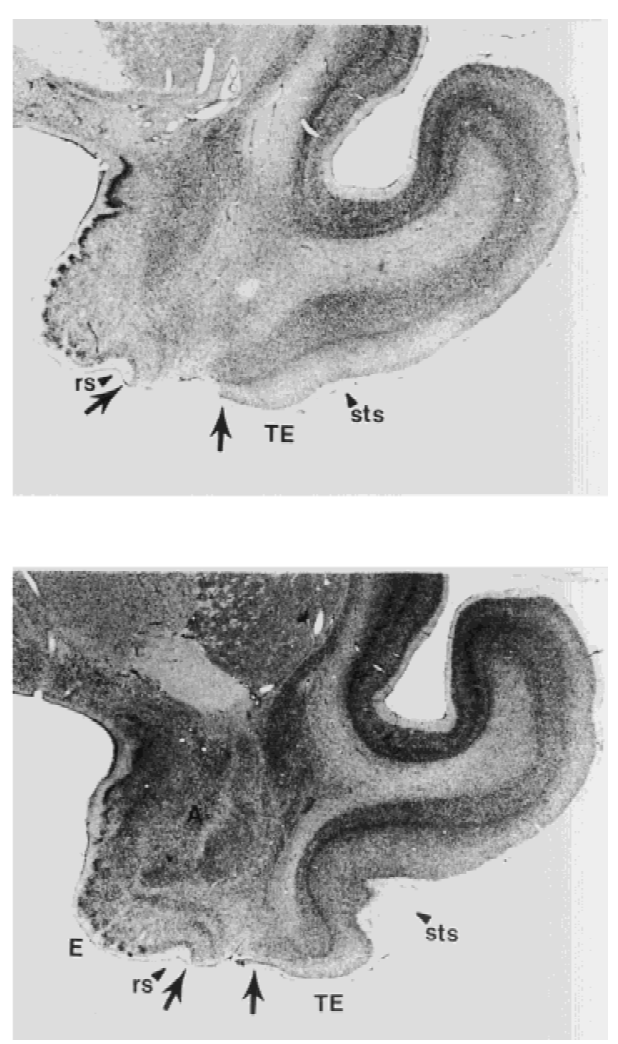

monkey PR 2 was estimated to have damaged $87 \%$ of the perirhinal cortex. There was no damage to either laterally adjacent area TE or the entorhinal cortex. The hippocampus and amygdala were completely spared bilaterally. Additionally, there was minimal white matter damage.

PR 3

Overall, the lesion in monkey PR 3 was estimated to have damaged $65 \%$ of the perirhinal cortex. Anterior to the amygdala, the damage to the

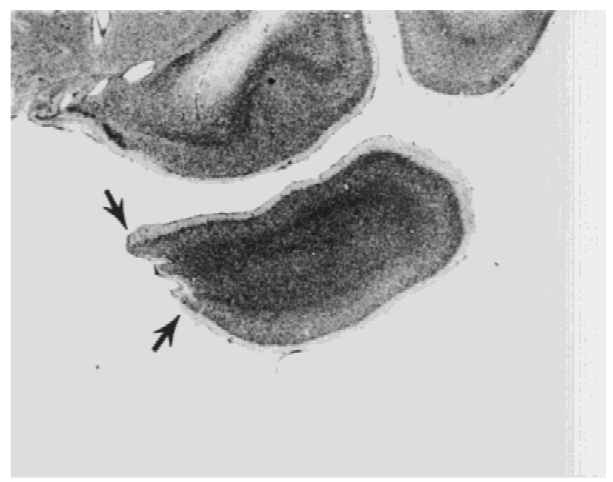

$$
\begin{array}{llllllllllllllll} 
& E & A & R & N & I & N & G & \boldsymbol{Q} & M & E & M & O & R & Y \\
\mathbf{5 8 2} & & & & & &
\end{array}
$$



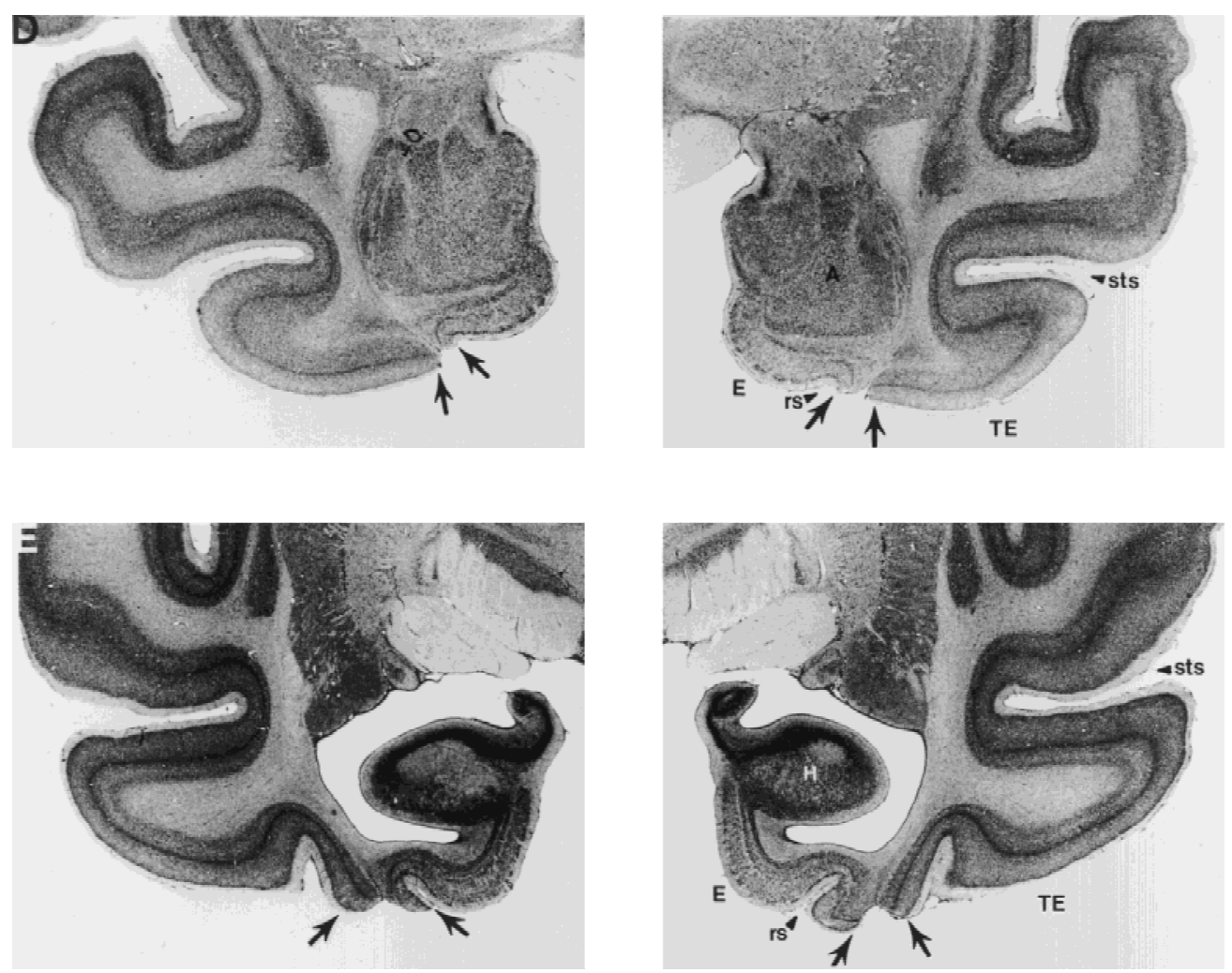

Figure 4: Photomicrographs of representative sections through the left and right temporal lobes of monkey PR 2, whose lesion most closely approximated the intended lesion (see Fig. 2). The sections are arranged from rostral $(A)$ to caudal $(E)$, and the lesion is indicated by arrows at each level. (rs) Rhinal sulcus; (sts) superior temporal sulcus; (TE) area TE; (A) amygdala; (E) entorhinal cortex; $(\mathrm{H})$ hippocampus.

perirhinal cortex was moderate, sparing most of the dorsal portion of area 36 and area 35 . From the level of the amygdala through the caudal extent of the perirhinal cortex, the damage to the perirhinal cortex was similar to that in monkey PR 2. At this level the damage was extensive, including both areas 35 and 36 . There was minimal damage to the most medial portion of area TE bilaterally, at the tip of the anterior middle temporal sulcus, extending posteriorly for $<1 \mathrm{~mm}$. Previously, we rated the amount of damage in lesioned brains on a scale from 0-3 (Buffalo et al. 1998b). A score of 0 corresponded to no damage, 1 corresponded to minimal (up to $\sim 35 \%$ ) damage, 2 corresponded to moderate (up to $\sim 65 \%$ ) damage and 3 corresponded to extensive (up to 100\%) damage. The amount of TE damage in monkey PR3 corresponds to a rating of 0.25 on the right and 0.02 on the left according to the scale used in Buffalo et al. (1998b). The lesion included moderate damage to the right parahippocampal cortex extending $\sim 4 \mathrm{~mm}$ into the parahippocampal cortex. The entorhinal cortex was com- pletely spared bilaterally. Additionally, the amygdala and hippocampal region were completely spared bilaterally. There was minimal white matter damage.

PR 4

The lesion of the perirhinal cortex in monkey PR 4 was estimated to have damaged $69 \%$ of the perirhinal cortex. There was moderate damage to the perirhinal cortex bilaterally starting from the pole, with more extensive damage on the right side. Similar to the lesion in monkey PR 2, at levels rostral to the amygdala, most of the dorsal portion of area 36 and area 35 was spared bilaterally. At the level of the amygdala, most of the perirhinal cortex was removed, including both areas 35 and 36 . The lesion extended through the caudal extent of the perirhinal cortex and included minimal damage on the left and moderate damage on the right to the medial portion of parahippocampal cortex extending about $3 \mathrm{~mm}$ in the rostrocaudal plane. Area TE

$$
\begin{array}{lllllllllllllll} 
& E & A & R & N & I & N & G & \mathbf{Q} & M & E & M & O & R & Y \\
\mathbf{5 8 3} & & & & & & &
\end{array}
$$




\section{Buffalo et al.}

and the entorhinal cortex were completely spared bilaterally. Neither the amygdala nor the hippocampal region was involved in the lesion, and there was little or no damage to the underlying white matter.

PR 5

The lesion of the perirhinal cortex in monkey PR 5 was estimated to have damaged $77 \%$ of the perirhinal cortex. There was extensive damage to the perirhinal cortex on the right side and moderate damage on the left in the polar region, although there was sparing of the dorsal portion of area 36 and area 35 rostral to the amygdala. From the level of the amygdala to the caudal extent of the perirhinal cortex, the lesion included both areas 35 and 36. There was a small amount of damage to area TE on the left side anterior to the level of the amygdala that extended $\sim 1.5 \mathrm{~mm}$ in the rostrocaudal plane, and there was a very small amount of damage to area TE on the left side at the level of the caudal amygdala. On the right side, there was a very small amount of damage to area TE that extended $\sim 1 \mathrm{~mm}$ in the rostrocaudal plane. This amount of TE damage corresponds to a rating of 0.22 on the left and 0.02 on the right, according to the scale used in Buffalo et al. (1998b). Additionally, on the left side, the lesion included minimal damage to the rostral parahippocampal cortex that extended $\sim 3.5 \mathrm{~mm}$ into the parahippocampal cortex. There was no damage to either area TE or the parahippocampal cortex on the right side. The entorhinal cortex, amygdala, and hippocampal region were completely spared bilaterally. There was minimal white matter damage.

PR 1

Although the damage to the perirhinal cortex in monkey PR 1 was similar to that in monkey PR 2, monkey PR 1 sustained an extensive amount of damage outside the intended lesion. The lesion was estimated to have damaged $73 \%$ of the perirhinal cortex. Similar to the other cases, the removal of the perirhinal cortex anterior to the amygdala was moderate in that it spared the dorsal portion of area 36 and area 35. At the level of the amygdala and through the caudal extent of the lesion, the perirhinal cortex removal was extensive, including both areas 35 and 36 . On the right side, there was no damage to area TE, and there was minimal damage to the parahippocampal cortex extending $\sim 2.5$ $\mathrm{mm}$ in the rostrocaudal plane. The entorhinal cor- tex, amygdala, and hippocampal region were completely spared bilaterally. There was minimal white matter damage.

When the brain of monkey PR 1 was removed, it was noticed that the left mandibular bone had protruded through the craniotomy that was made during surgery, and this protruding bone had compressed most of the middle temporal gyrus on the left side. This protrusion caused an extensive amount of direct damage to area TE unilaterally, as well as extensive compression of the remaining intact area TE tissue on that side. The damage to area TE extended from the pole through the caudal extent of area TE. This protrusion also caused a minimal amount of compression of the rostral parahippocampal cortex extending for $\sim 4 \mathrm{~mm}$ in the rostrocaudal plane. Because the histological analysis of the lesion in this monkey was carried out $\sim 5$ years after the surgical removal of the perirhinal cortex, it is impossible to determine exactly when this protrusion occurred during this 5 -year period. That is, it is unclear when, during the course of behavioral testing, the unilateral damage to area TE occurred. The overall behavioral findings did not change when the scores of monkey PR 1 were excluded.

HISTOLOGICAL RESULTS: DESCRIPTIONS

OF THE LESIONS IN THE TE GROUP

GENERAL OBSERVATIONS

For each of the lesions in the TE group, the extent of removal will be described at four rostrocaudal levels: at the level of the region rostral to the amygdala, corresponding to A19.0 in Figure 2; at the level of the amygdala, corresponding to A16.4 in Figure 2; at the level of the rostral hippocampus, corresponding to A13.4 in Figure 2; and at the caudal extent of area TE, corresponding to A6.6 in Figure 2. On average, the TE group sustained an estimated $61 \%$ damage to area TE (TE $1=44 \%$, TE $2=55 \%$, TE $3=69 \%$, TE $4=64 \%$, TE $5=71 \%$. On average, the TE group sustained an estimated $32 \%$ damage to the portion of area TE that lies in the ventral bank of the superior temporal sulcus. All monkeys sustained moderate to extensive removal of area TE throughout the rostrocaudal extent of area TE. The amount of unintended damage outside area TE was minimal in all cases. In all cases there was little or no damage to the perirhinal cortex. However, monkey TE 5 sustained minimal bilateral compression of the peri-

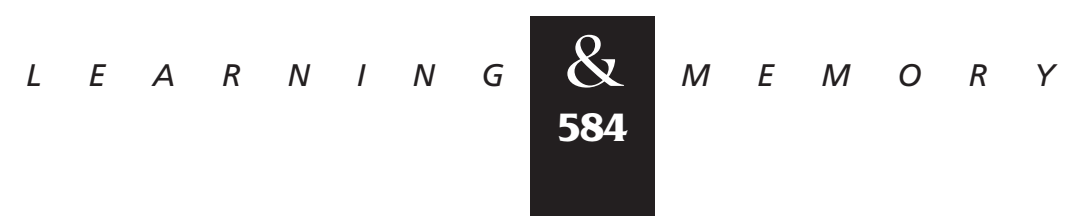


rhinal cortex extending $\sim 2 \mathrm{~mm}$ on the left and $\sim 1$ $\mathrm{mm}$ on the right. This compression was due to extensive enlargement of the ventricles at the level of the caudal amygdala and the hippocampus. It is not clear whether this ventricle enlargement was accompanied by direct damage to the white matter at these levels. The parahippocampal cortex was spared in all cases except for monkeys TE 2 and TE 5, who sustained a minimal amount of damage to the parahippocampal cortex. The rostral portion of area TEO was minimally damaged in all five TE monkeys. Additionally, in no cases was there damage to the entorhinal cortex, the amygdala, or the hippocampal region. There was minimal white matter damage in all but two cases (TE 3 and TE 5).

The extent of the lesion in each monkey is plotted on representative coronal sections (Fig. 5). The rostrocaudal levels used to illustrate the lesions are the same as those used in Figure 2. The full rostrocaudal extent of the lesion in animal TE 4 is shown in Figure 6. The lesion in this monkey most closely approximated the intended lesion and is described first. Then, shorter, comparative descriptions of the lesions in the other animals in the TE group are provided.

TE 4

At its rostral extent, the lesion in monkey TE 4 began bilaterally just at the tip of the STS (A22.0; Fig. 6), with damage involving only cortical layers I and II. Just rostral to the amygdala (A19.0; Fig. 6), the lesion expanded to encompass all layers of area TE. At this level, however, there was some sparing of medial portions of area TE. Additionally, most of the ventral bank of the STS was spared on the left. At the level of the amygdala (A16.4; Fig. 6), there was moderate removal of area TE, with the AMTS completely spared bilaterally. There was no damage to laterally adjacent perirhinal cortex. Additionally, there was minimal damage to the underlying white matter. At the level of the hippocampus (A13.4; Fig. 6), the removal of area TE continued to spare portions of the ventral bank of the STS. In addition, laterally adjacent parahippocampal cortex was completely spared bilaterally. At this level there was moderate damage to the underlying white matter. The removal of area TE continued through the caudal extent of area $\mathrm{TE}$ (A6.6; Fig. 6) and included extensive damage on the left and moderate damage on the right to the underlying white matter. At caudal levels, the lesion included minimal damage to the rostral por- tion of area TEO for $\sim 2 \mathrm{~mm}$ on the left and $0.5 \mathrm{~mm}$ on the right. The lesion was estimated to have damaged $64 \%$ of area TE. Finally, the lesion was estimated to have damaged $42 \%$ of the portion of area TE that lies in the ventral bank of the STS.

TE 1

The removal of area TE in monkey TE 1 was less complete than in monkey TE 4 and was estimated to have damaged $44 \%$ of area TE. Although there was moderate damage to area TE throughout its rostrocaudal extent, the AMTS was completely spared bilaterally. The lesion was estimated to have damaged $14 \%$ of the portion of area TE that lies in the ventral bank of the STS. The perirhinal cortex was completely spared bilaterally. On the left side there was minimal damage to the parahippocampal cortex that extended $\sim 1.0 \mathrm{~mm}$ in the rostrocaudal plane. The parahippocampal cortex was completely spared on the right side. The lesion included minimal damage to the rostral portion of area TEO, extending for $\sim 3.0 \mathrm{~mm}$ in the rostrocaudal plane, bilaterally. There was minimal damage to the underlying white matter, which was more prominent on the left side than on the right.

TE 2

The removal in monkey TE 2 was estimated to have damaged $\sim 55 \%$ of area TE. The lesion was estimated to have damaged $42 \%$ of the portion of area TE that lies in the ventral bank of the STS. Rostral to the amygdala, the lesion was extensive, involving most of area TE including the tissue in the ventral bank of the STS. At the level of the amygdala, part of the ventral bank of the STS was spared bilaterally. There was minimal sparing of the most medial portion of area TE. There was minimal damage to the parahippocampal cortex that extended for $\sim 2.5 \mathrm{~mm}$ in the rostrocaudal plane, bilaterally. The lesion continued through the caudal extent of area TE on the left side; there was some caudal sparing of area TE $(\sim 2.0 \mathrm{~mm})$ on the right side. Area TEO was damaged minimally on the left, extending $\sim 2.0 \mathrm{~mm}$ into area TEO. There was no damage to the perirhinal cortex, amygdala, or hippocampal region, and there was minimal white matter damage throughout the extent of the lesion.

TE 3

The removal in monkey TE 3 was estimated to have damaged $69 \%$ of area TE. The lesion was es-

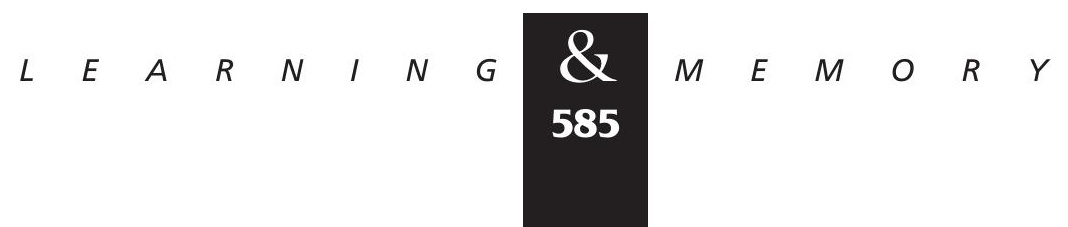




\section{Buffalo et al.}

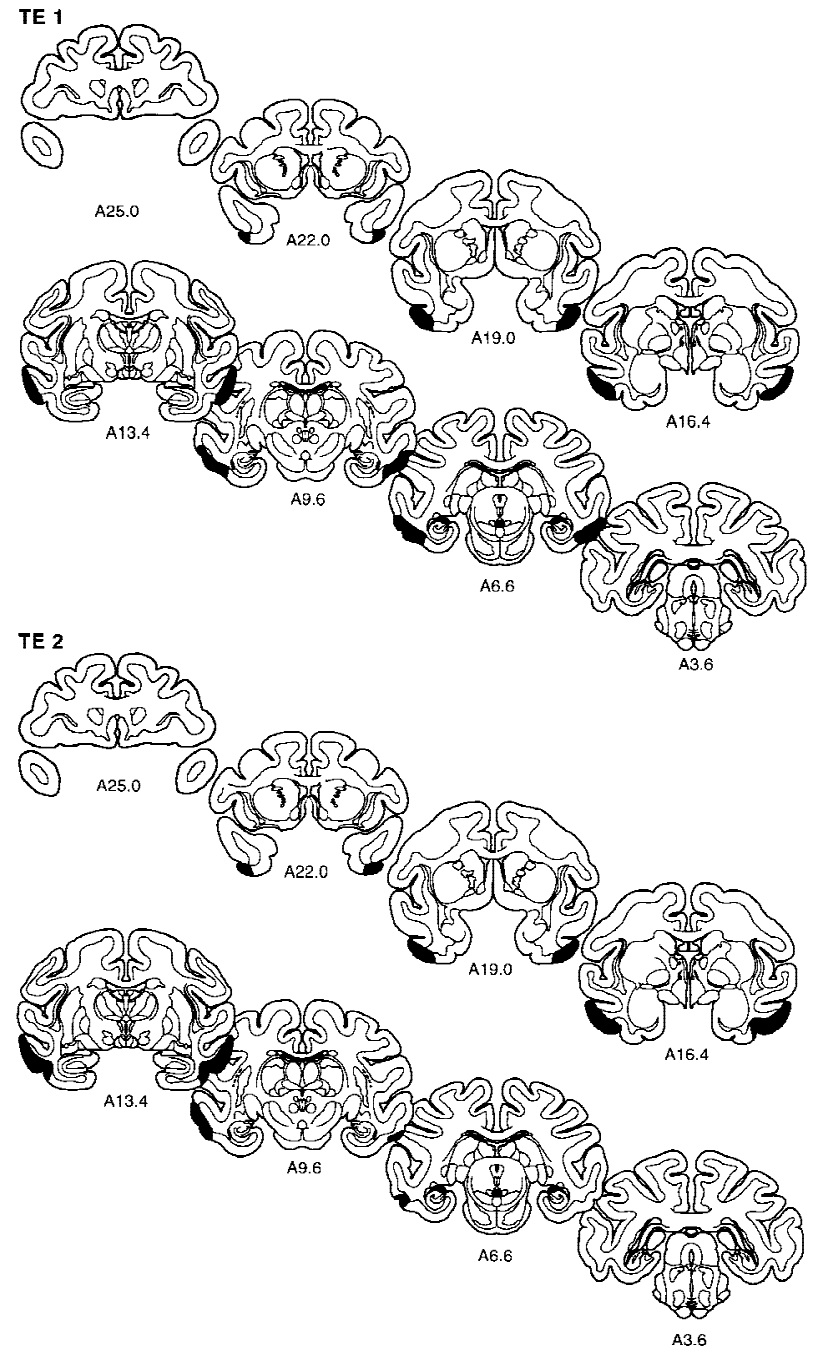

Figure 5: The extent of the lesions of area TE in each of the five monkeys in the TE group (TE 1-TE 5) is plotted on representative coronal sections redrawn from the atlas of Szabo and Cowan (1984). In each case, the area of the lesion is indicated in black. The rostrocaudal level is indicated below each section (see Figs. 1 and 2 for borders of area TE).

timated to have damaged $27 \%$ of the portion of area TE that lies in the ventral bank of the STS. Anteriorly, the lesion began at about the tip of the STS. On the right side, the removal included the

TE 3

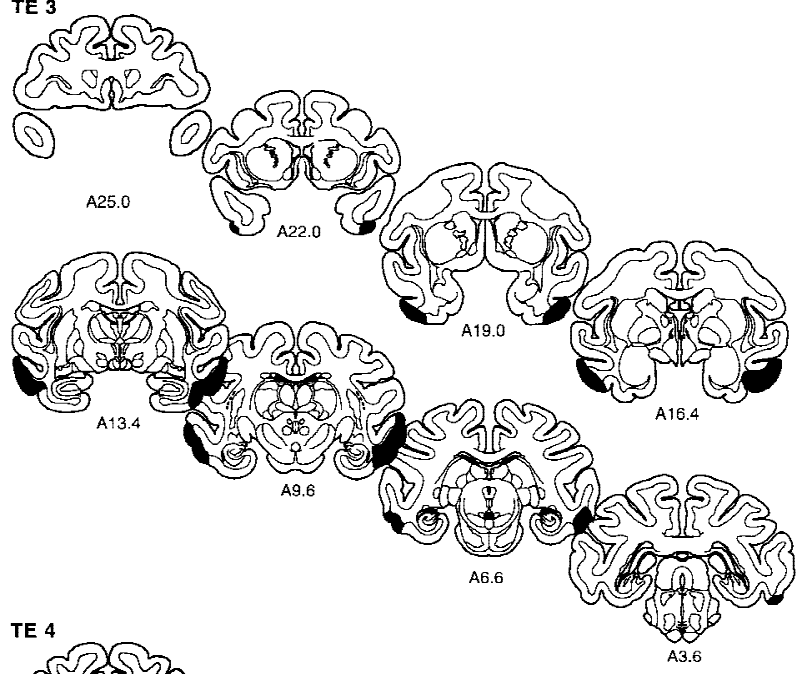

(0)

(1)
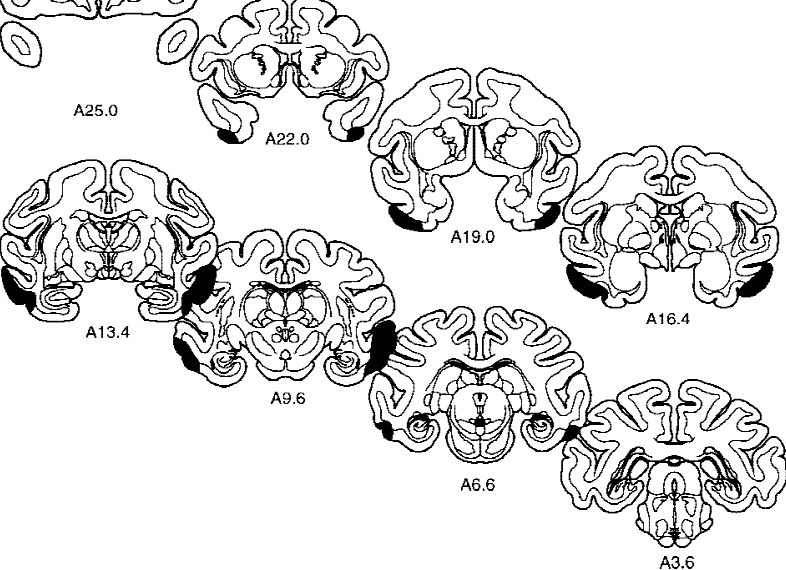

ventral bank of the STS, but this tissue was spared on the left side. There was some sparing of medial area TE tissue bilaterally. At the level of the rostral amygdala, the removal was fairly extensive and in-

$$
\begin{array}{lllllllllllllll} 
& E & A & R & N & I & N & G & \mathbf{8} \\
\mathbf{5 8 6} & M & E & M & 0 & R & Y
\end{array}
$$


cluded all of area TE except the portion that lies in the ventral bank of the STS on the left side. At the level of the caudal amygdala, the removal was more moderate, with bilateral sparing of tissue in the ventral bank of the STS and in both banks of the AMTS on the left side. The lesion continued through the caudal extent of area TE and into rostral area TEO. Area TEO was minimally damaged on the right, extending $\sim 5.5 \mathrm{~mm}$. Bilaterally, the perirhinal cortex, the parahippocampal cortex, the amygdala, and the hippocampal region were completely spared. There was moderate damage to the underlying white matter on the left side that extended $\sim 6 \mathrm{~mm}$ in the rostrocaudal plane. Again, the damage on the right side was more extensive, with the white matter damage extending $\sim 11.5 \mathrm{~mm}$ in the rostrocaudal plane.

TE 5

The removal in monkey TE 5 was estimated to have damaged $71 \%$ of area TE. The lesion was estimated to have damaged $35 \%$ of the portion of area TE that lies in the ventral bank of the STS. The lesion began anterior to the tip of the STS on the left side and at the tip of the STS on the right side. At levels rostral to the amygdala, the damage to area TE was extensive and included the ventral bank of the STS. From the level of the amygdala through the caudal extent of area TE, the damage to area TE was more moderate, with sparing of tissue laterally, in the ventral bank of the STS, as well as at medial aspects of area TE. Additionally, at the level of the mid-amygdala, the ventricles began to enlarge bilaterally. This enlargement continued and expanded through the caudal amygdala and through the hippocampal region. This ventricle enlargement caused compression of the lateral perirhinal cortex and parahippocampal cortex bilaterally. The compression of the perirhinal cortex extended in the rostrocaudal plane $\sim 2.0 \mathrm{~mm}$ on the left side and $\sim 1.0 \mathrm{~mm}$ on the right side. The compression of the parahippocampal cortex extended in the rostrocaudal plane $\sim 3.0 \mathrm{~mm}$, bilaterally. Additionally, there was some very minimal rostral area TEO damage extending $\sim 1.5 \mathrm{~mm}$ into area TEO on the left side and $2.0 \mathrm{~mm}$ into area TEO on the right side. The ventricle enlargement did not cause any direct damage to the amygdala or the hippocampus. However, there was some displacement of the lateral nucleus of the amygdala bilaterally. There was also moderate white matter damage, bilaterally, extending from the level of the cau- dal amygdala through the caudal extent of the lesion.

\section{Experiment 1}

\section{Results}

V-DNMS

Figure $7 \mathrm{~A}$ shows the mean number of trials required by each group of monkeys to reach criterion level performance on the first administration of the V-DNMS task with a delay of $8 \mathrm{sec}$. Individual scores for each monkey in the PR, TE, and $\mathrm{N}$ groups are shown in Figure 7A and Table 1. Although the PR and TE groups required numerically more trials on average to obtain criterion level performance (620 trials and 670 trials, respectively, compared with 196 trials for the $\mathrm{N}$ group), a oneway ANOVA comparing the performance of the three groups of monkeys revealed no significant effect of group $[F(2,17)=2.43, P>0.10]$. On the second administration of the V-DNMS task with a delay of $8 \mathrm{sec}$, a one-way ANOVA comparing the performance of the three groups of monkeys revealed a significant effect of group $[F(2,17)=5.50$, $P<0.05]$. Separate comparisons of each group's performance revealed that the PR group was significantly impaired relative to the $\mathrm{N}$ group ( $\mathrm{PR}=228$ trials, $\mathrm{N}=12$ trials; $P<0.05$ ). The performance of the TE group was not different from either the $\mathrm{N}$ group or the PR group (TE $=44$ trials; both $P$ s $>0.10$ ).

Figure $7 \mathrm{~B}$ shows performance of the three groups of monkeys across delays on the two DNMS tests, averaged together for each monkey.The score for the 8-sec delay is the average score on the final 100 trials of training for the two times that the task was given. A two-way ANOVA involving all three groups and four delays (15 sec, $1 \mathrm{~min}, 10$ $\mathrm{min}$, and $40 \mathrm{~min}$ ) revealed a significant effect of group $[F(2,17)=14.32, P<0.01$; see Fig. $7 \mathrm{C}]$ and a significant effect of delay $[F(3,51)=118.90$, $P<0.01]$, but no group by delay interaction $[F$ $(6,51)=0.72, P>0.10]$. Post-hoc analysis (Fisher's PLSD, two-tailed) revealed that both operated groups were impaired relative to the $\mathrm{N}$ group (both Ps $<0.01$; see Fig. 7C).

Separate comparisons (two-tailed $t$-tests) based on each group's scores at each of the delays revealed that the $\mathrm{PR}$ and $\mathrm{TE}$ groups performed significantly worse than the $\mathrm{N}$ group at the 15sec delay $(\mathrm{N}=94 \%, \mathrm{PR}=85 \%, \mathrm{TE}=89 \%$; both

$$
\begin{array}{lllllllllllllll}
L & E & A & R & N & I & N & G & \underset{\mathbf{5 8 7}}{\boldsymbol{Z}} & M & E & M & O & R & Y
\end{array}
$$




\section{Buffalo et al.}

$P$ s $<0.01)$; at the $1-\mathrm{min}$ delay $(\mathrm{N}=91 \% ; \mathrm{PR}=83 \%$, $P<0.01 ; \mathrm{TE}=86 \%, P=0.05) ;$ and at the $10-\mathrm{min}$ delay $(\mathrm{N}=77 \% ; \quad \mathrm{PR}=69 \%, \quad P<0.05 ; \quad \mathrm{TE}=69 \%$, $P=0.05)$. At the $40-$ min delay, the TE group performed significantly worse than the $\mathrm{N}$ group (N $=72 \%$; $\mathrm{TE}=63 \%, P<0.01)$. The PR group performed numerically worse than the $\mathrm{N}$ group, but this difference did not reach statistical significance (PR $=66 \%, P=0.09$ ). The PR and TE groups performed similarly at all delays (all $P$ s $>0.10$ ). Scores for each monkey on the two separate presentations of the V-DNMS task are given in Table 1. The over-
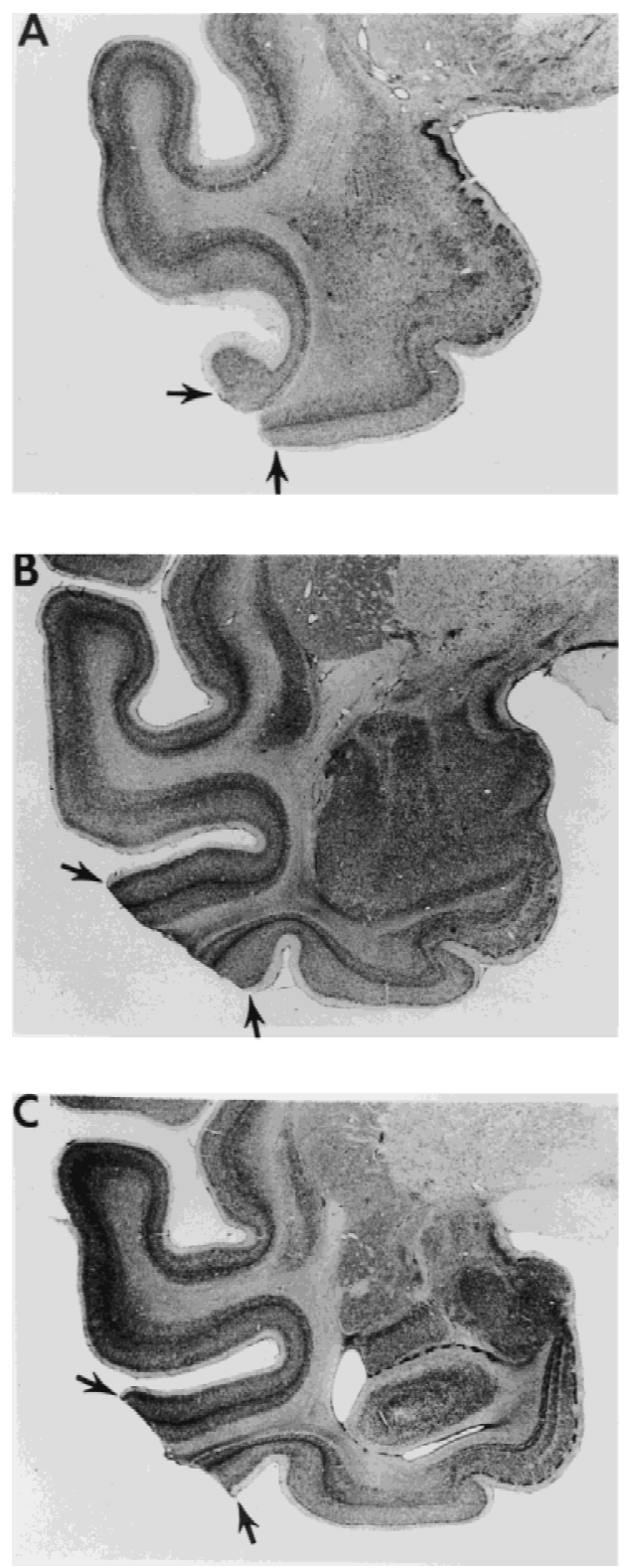

all findings were the same when each presentation was analyzed separately.

In summary, compared with the $\mathrm{N}$ group, the PR group and the TE group exhibited an overall deficit on the delay portion of the V-DNMS task. The two lesion groups performed similarly.

T-DNMS

There were no group differences in the amount of time required to complete any of the tactual pretraining phases. Figure $8 \mathrm{~A}$ shows the
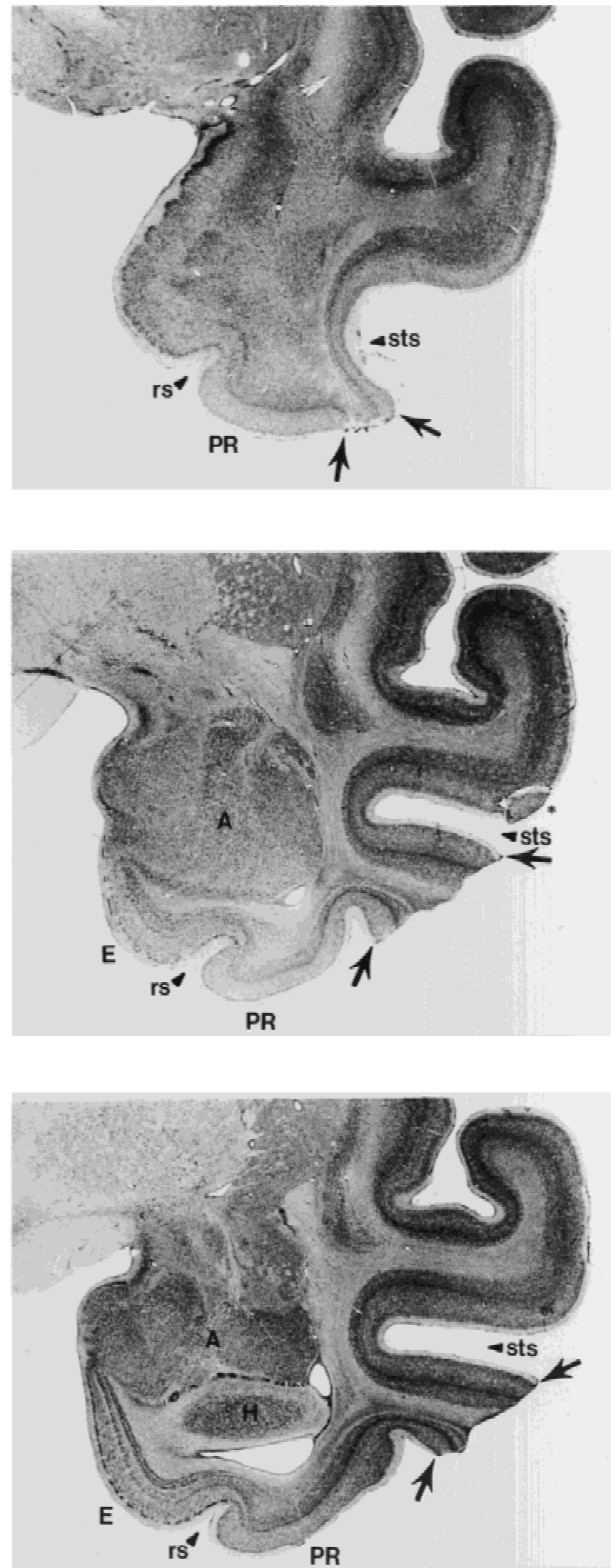

Figure 6: (Continued on facing page.)

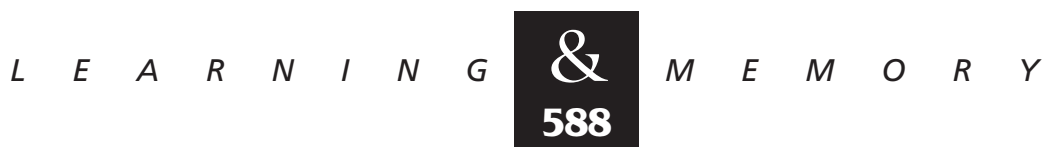



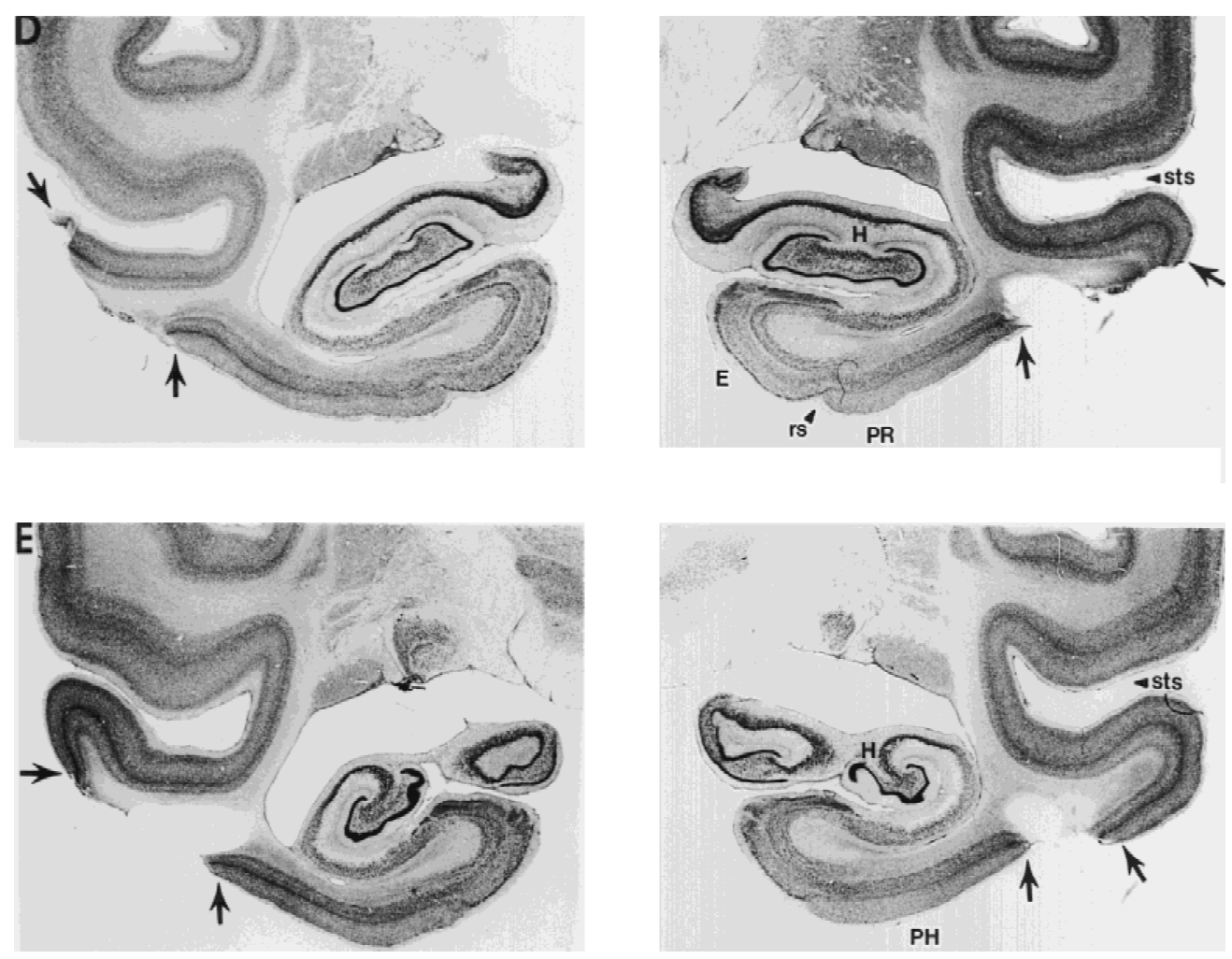

Figure 6: Photomicrographs of representative sections through the left and right temporal lobes of monkey TE 4, whose lesion most closely approximated the intended lesion (see Fig. 2). The sections are arranged from rostral $(A)$ to caudal $(E)$, and the lesion is indicated by arrows at each level. The asterisk $(*)$ indicates a processing artifact. (rs) Rhinal sulcus; (sts) superior temporal sulcus; (PR) perirhinal cortex; (E) entorhinal cortex; (A) amygdala; (H) hippocampal region; (PH) parahippocampal cortex.

mean number of trials required by each group of monkeys to reach criterion level performance on the basic task with an 8-sec delay. Individual scores for the PR, TE, and $\mathrm{N}$ groups are shown in Figure 8A and in Table 1. A one-way ANOVA comparing the performance of the three groups of monkeys revealed a significant effect of group $[F(2,8)=8.06, P<0.05]$. Post-hoc analysis (Fisher's PLSD, two-tailed) revealed that the PR group was significantly impaired relative both to the $\mathrm{N}$ group and to the TE group ( $\mathrm{PR}=561$ trials, $\mathrm{TE}=184$ trials, $\mathrm{N}=380$ trials, $P \mathrm{~s}<0.05)$. The TE group was unimpaired relative to the $\mathrm{N}$ group $(P>0.05)$. Figure $8 \mathrm{~B}$ shows the performance of the three groups across delays from $8 \mathrm{sec}$ to $10 \mathrm{~min}$. A two-way ANOVA involving the three groups (N, PR, and TE) and the three longer delays $(15 \mathrm{sec}, 1 \mathrm{~min}$, and $10 \mathrm{~min}$ ) revealed a significant effect of group $[F(2,8)=50.08, P<0.01]$ and delay $[F(2,16)=74.43, P<0.01]$ and no significant group $\times$ delay interaction $[F(2,16)=3.25$, $P>0.05]$.
Separate comparisons (two-tailed $t$-tests) based on each group's scores at each of the delays revealed that the PR group performed significantly worse than both the TE group and the $\mathrm{N}$ group at the 15 -sec delay $(\mathrm{N}=92 \%, \mathrm{TE}=89 \%, \mathrm{PR}=77 \%$; PR vs. N, $P<0.01$; PR vs. TE, $P<0.05)$, at the 1 - min delay $(\mathrm{N}=85 \%, \mathrm{TE}=83 \%, \mathrm{PR}=69 \%$; $\mathrm{PR}$ vs. N, $P<0.01$; PR vs. TE, $P<0.05)$, and at the 10 -min delay $(\mathrm{N}=75 \%$; $\mathrm{TE}=70 \%$; $\mathrm{PR}=47 \%$ both $P$ s $<0.01)$. The TE group performed similarly to the $\mathrm{N}$ group at all delays (all $P \mathrm{~s}>0.10$ ).

Figure 8C shows the performance of the three groups of monkeys averaged across all three delays (15 sec, $1 \mathrm{~min}$, and $10 \mathrm{~min}$ ). The PR group was significantly impaired relative to the $\mathrm{N}$ and TE groups $(\mathrm{N}=84 \%, \mathrm{TE}=81 \%, \mathrm{PR}=64 \%$; both $P$ s $<0.01)$. The TE group performed similarly to the $\mathrm{N}$ group $(P>0.10)$.

In summary, the results from the T-DNMS task demonstrate a dissociation between the PR and TE groups. Compared with both the $\mathrm{N}$ group and the TE group, the PR group exhibited a severe deficit

$$
\begin{array}{lllllllllllllll}
\hline & E & A & R & N & I & N & G & \mathbf{Q} \\
\mathbf{5 8 9} & M & E & M & O & R & Y
\end{array}
$$




\section{Buffalo et al.}
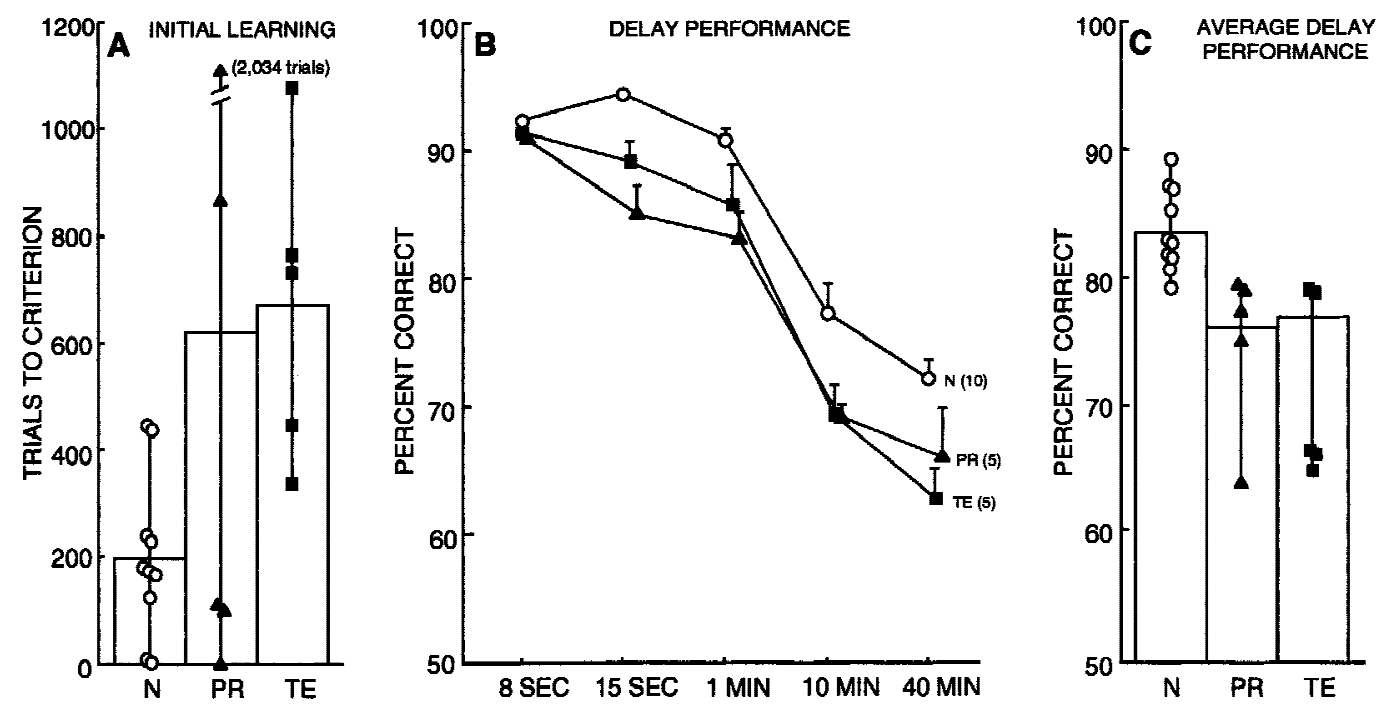

Figure 7: Performance on the $\mathrm{V}$-DNMS task by normal monkeys $(\mathrm{N}=10)$, monkeys with lesions of the perirhinal cortex $(P R=5)$, and monkeys with lesions of area TE $(T E=5)$. (A) Performance on initial learning on the first administration of the V-DNMS task with an 8-sec delay. Symbols show trials to criterion for individual monkeys. (B) Performance across delays on the two administrations of the V-DNMS task, averaged together for each monkey. Bars represent S.E.M.. (C) Performance across all delays $(15 \mathrm{sec}, 1 \mathrm{~min}, 10 \mathrm{~min}$, and $40 \mathrm{~min}$ averaged together) on the two administrations of the V-DNMS task. Symbols show the performance of individual monkeys. See Table 1 for individual scores of all monkeys.

on the T-DNMS task (Fig. 8C). The TE group performed similarly to the $\mathrm{N}$ group on this task.

\section{Discussion}

The results from experiment 1 demonstrated a dissociation between the effects of lesions limited to the perirhinal cortex and lesions limited to area TE. Although both operated groups were similarly impaired on the test of visual recognition memory, these groups performed differently on the test of tactual recognition memory. Specifically, on the T-
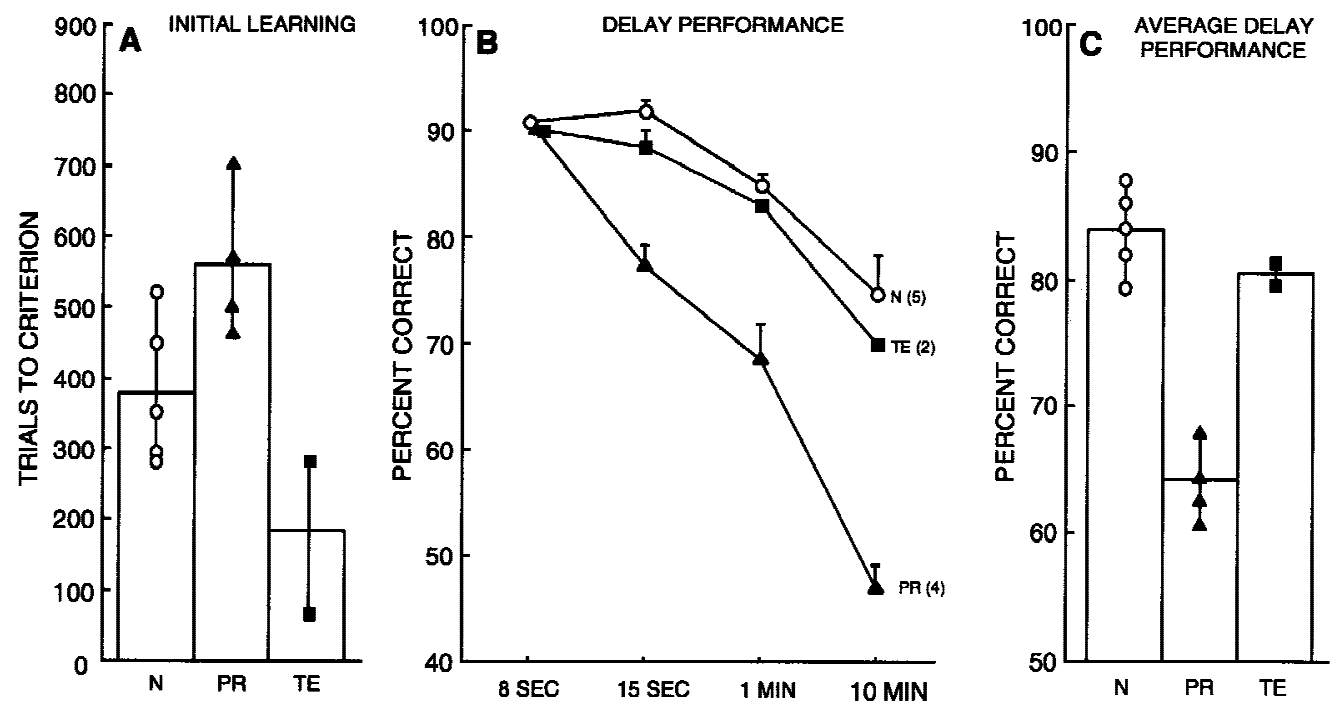

Figure 8: Performance on the T-DNMS task by normal monkeys $(N=5)$, monkeys with lesions of the perirhinal cortex $(\mathrm{PR}=4)$, and monkeys with lesions of area TE $(\mathrm{TE}=2)$. (A) Performance on initial learning of the T-DNMS with an 8-sec delay. Symbols show trials to criterion for individual monkeys. (B) Performance across delays on the T-DNMS task. Bars represent S.E.M. (C) Performance across all delays (15 sec, $1 \mathrm{~min}$, and $10 \mathrm{~min}$ averaged together) on the T-DNMS task. Symbols show the performance of individual monkeys. See Table 1 for individual scores of all monkeys.

$$
\begin{array}{lllllllllllllll}
\hline & A & R & N & I & N & G & \mathbf{8} \\
\mathbf{5} 90 & M & E & M & 0 & R & Y
\end{array}
$$


DNMS task, monkeys in the PR group were impaired both in learning the task with an 8-sec delay (Fig. 8A) and in performing the task at delays of 15 sec, $1 \mathrm{~min}$, and $10 \mathrm{~min}$ (Fig. 8B,C). In contrast, the TE group performed normally on the T-DNMS task. Monkeys and humans with damage to the medial temporal lobe are known to exhibit multimodal memory deficits (Milner 1972; Murray and Mishkin 1984; Suzuki et al. 1993; Malkova et al. 1995; Goulet and Murray 1996). The findings from the present study extend these findings by showing that lesions limited to the perirhinal cortex itself are sufficient to produce a severe deficit in both visual and tactual recognition memory in monkeys.

\section{Experiment 2: Do the Perirbinal Cortex and Area TE Make Distinct Contributions to Visual Recognition Memory?}

\section{Materials and Methods}

\section{SUBJECTS}

The 5 PR monkeys, 3 of the TE monkeys, and 13 of the control monkeys (N6-N18) were tested on the VPC task. Prior to the administration of the VPC task, two monkeys from the TE group (TE1 and TE 2) had to be euthanized as a result of gastrointestinal disorder.

BEHAVIORAL TESTING: VPC

PRETRAINING

Subjects were habituated for $20 \mathrm{~min}$ a day for 5 days to a primate chair that was in a darkened, well-ventilated, sound and light attenuating chamber $(78 \mathrm{~cm} \times 55 \mathrm{~cm} \times 46 \mathrm{~cm})$. The subjects faced a rear projection screen located $41 \mathrm{~cm}$ directly in front of the subject where the stimuli would be presented.

TESTING

First, the monkey viewed two identical stimuli side by side. The monkey was required to look at the stimuli for a cumulative looking time of $25 \mathrm{sec}$ (familiarization). Then, after a delay interval, the monkey again viewed two stimuli, the familiar stimulus and a new stimulus for a total of $5 \mathrm{sec}$ of cumulative looking. One second after the completion of the first test phase, a second test phase was presented. In the second test phase, which controlled for side preferences, the old stimulus was again presented with another new stimulus, but the left/right position of the old stimulus was reversed. Memory was assessed by the amount of time monkeys looked at the new stimulus. If a monkey looked longer at the new stimulus, it can be inferred that he had a memory for the familiar, and now less interesting, stimulus. Monkeys were given 10 trials a day. Each day, one of four delays was given. The delays were $1 \mathrm{sec}, 10 \mathrm{sec}, 1 \mathrm{~min}$, and $10 \mathrm{~min}$. The order of the delays was randomized for each subject.

During testing sessions, the position of the subject's eyes were recorded by a high resolution video camera (Sony Video Hi8 CCD-TR600) unobtrusively mounted just above the rear-projection screen. The video images were displayed on a Sony Trinitron superfine pitch TV monitor (model no. CUM-1271). The video images were also routed into a 16-bit Video VGA board where a computer program automatically overlaid trial information (trial number, test phase, and delay interval) onto the video image before storage to tape (JVC Super VHS). The experimenter controlled the length of the familiarization and test phases, and the computer controlled the delay interval.

The stimuli were high contrast, black and white computer-generated two-dimensional shapes. The stimuli were transferred to photographic slides that contained two stimuli each, $11 \times 18 \mathrm{~cm}$ in size and separated from each other by $11 \mathrm{~cm}$ when projected onto the screen. A Kodak carousel projector (model no. $760 \mathrm{H}$ ) was encased in a sound insulating box so that slides could be advanced silently. The projected stimuli were passed through a red filter (A25) to reduce the brightness of the background. Pilot work had indicated that without a filter, monkeys tended to avoid looking at the rear projection screen and the stimuli because of the intense light from the projector.

SCORING

Each session's videotape was scored by two experimenters blind to the test conditions. The position of the eye (i.e., fixating the left or right stimulus on the screen) was determined by analyzing the position of reflected light off the cornea. If the reflection was on the left side of the pupil, the subject was looking right, and if the reflection was on the right side of the pupil, the subject was looking left. During pilot work we were able to con-

$$
\begin{array}{lllllllllllllll}
L & E & A & R & N & I & N & G & \mathbf{Q} & M & E & M & O & R & Y \\
\mathbf{5 9 1} & & & & & &
\end{array}
$$




\section{Buffalo et al.}

struct precise descriptions of what constituted stimulus fixation and what constituted looking between or to the side of the stimuli. Scoring was accomplished by counting the number of frames (frame-by-frame analysis) in which the subject looked at the right or left stimulus. Overall interrater-reliability was 0.94 . The left and right frame counts were then entered into a computer along with a "familiar" versus "new" scoring key. The computer converted the frame counts into percentages of looking right versus left for each test phase, decoded the familiar versus new scoring key, and determined the percent preference for the new stimulus for each individual trial and for the entire session.

\section{Results}

VPC

Figure 9 and Table 1 show the performance of the three groups ( 3 TE monkeys, 5 PR monkeys, and $13 \mathrm{~N}$ monkeys) across the delays of $1 \mathrm{sec}$, $10 \mathrm{sec}, 1 \mathrm{~min}$, and $10 \mathrm{~min}$. A two-way ANOVA using all groups and all delays revealed a significant effect of group $[F(2,18)=17.07, P<0.01]$, and significant effect of delay $[F(3,54)=2.94$, $P<0.05$ ] but no significant group $\times$ delay interaction $[F(6,54)=1.62, P>0.10]$.

Individual comparisons (two-tailed $t$-tests) were performed to determine differences between

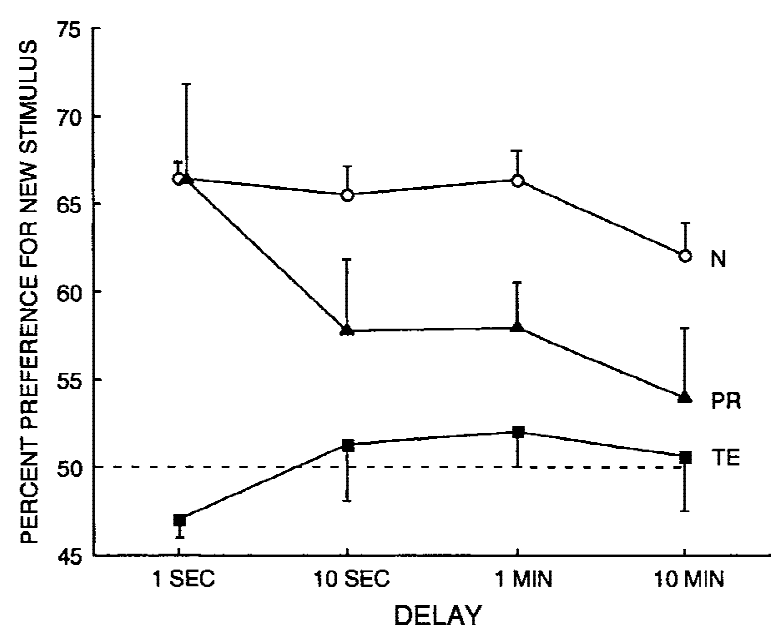

Figure 9: Performance on the VPC task by normal monkeys $(N=13)$, monkeys with lesions of the perirhinal cortex $(P R=5)$, and monkeys with lesions of area TE $(T E=3)$. Bars represent S.E.M. Broken line represents chance performance. the groups at each of the four delays. The TE group was impaired even at the shortest (1-sec) delay. That is, the monkeys with TE lesions looked at the novel stimulus only $47.0 \%$ of the time, whereas the $\mathrm{N}$ monkeys looked at the novel stimulus $66.4 \%$ of the time $(P<0.01)$. Additionally, at the 1 -sec delay, the TE group did not perform significantly above chance $(P>0.10)$. In contrast, the PR group was unimpaired at the 1 -sec delay $(\mathrm{PR}=66.4 \%$ preference, $P>0.10$ ). Moreover, at the 1 -sec delay, the TE group performed significantly worse than the PR group $(P<0.01)$. Importantly, the finding of intact performance by the PR group at the 1 -sec delay indicates that the PR group, unlike the TE group, did not have any primary deficit in perception or in the appreciation of novelty.

At the 10-sec delay, both the TE and PR groups were impaired relative to the $\mathrm{N}$ group $(\mathrm{TE}=51.3 \%$ preference, $\mathrm{PR}=57.8 \%$ preference, $\quad \mathrm{N}=65.5 \%$ preference; TE versus N, $P<0.05 ; \mathrm{PR}$ vs. N, $P<0.05)$. At the 1 -min delay, both operated groups were impaired relative to the $\mathrm{N}$ group (TE $=52.0 \%$ preference, $\mathrm{PR}=58.0 \%$ preference, $\mathrm{N}=66.3 \%$ preference; TE vs. $\mathrm{N}, P<0.01$; PR vs. $\mathrm{N}$, $P<0.05)$. At the 10 -min delay, both operated groups were impaired relative to the $\mathrm{N}$ group ( $\mathrm{TE}=50.6 \%$ preference, $\mathrm{PR}=54.0 \%$ preference, $\mathrm{N}=62.1 \%$ preference; both $P$ s $<.05)$. Additionally, there was no difference between the two operated groups at any of the delays of $10 \mathrm{sec}$ and longer (all $P$ s $>0.10$ ). Finally, at these longer delays, the TE monkeys did not perform above chance $[t s(3)<1.0, P s>0.10]$. The PR monkeys performed above chance at the 1-min delay $[t(4)=3.16, P<0.05]$ but not at $10 \mathrm{sec}$ or $10 \mathrm{~min}$ $[t \mathrm{~s}(4)<1.93, P \mathrm{~s}>0.10]$.

The amount of time needed to accumulate 25 sec of looking time during the familiarization phase was similar among the N, PR, and TE groups (27.2, 29.1, and $31.5 \mathrm{sec}$, respectively; one-way ANOVA, $P>0.10$ ). The TE group required a little more time than the $\mathrm{N}$ group to accumulate $25 \mathrm{sec}$ of looking time (N vs. TE, $P<0.05$; PR vs. TE, $P>0.10$ ). However, there was no suggestion of a gross abnormality in viewing behavior.

In summary, the findings point to a dissociation between the TE and PR groups. Specifically, at the shortest delay tested ( $1 \mathrm{sec}$ ), the TE group was significantly impaired, whereas the PR group performed normally. Additionally, at this delay the TE group performed significantly worse than the PR group. Moreover, the PR group was not impaired until after a delay of $10 \mathrm{sec}$ or longer. In contrast, 
the TE group was impaired at all delays; the performance of the TE group was not different from chance at any delay tested.

\section{Discussion}

The findings from experiment 2 provide additional evidence of a dissociation between the effects of perirhinal cortex lesions and area TE lesions. Lesions of the perirhinal cortex selectively impaired long-term memory. Immediate memory was intact. The impairment in the PR group cannot be attributed to a visual perceptual deficit, because such a deficit would be expected to impair performance at the short delay as well as at longer delays. In contrast, the TE group was severely impaired even at a delay as short as $1 \mathrm{sec}$. This impairment suggests that the TE group could not adequately perceive, attend to, or process the visual stimuli. In summary, the pattern of results indicates that the deficits following lesions of the perirhinal cortex and lesions of area TE are distinct. The impairment following lesions of the perirhinal cortex is best described as a memory impairment, whereas the impairment following lesions of area TE is best described as a visual perceptual deficit.

Experiment 3: Do the Perirbinal Cortex and Area TE Make

Distinct Contributions to Visual Discrimination Learning?

\section{Materials and Methods}

\section{SUBJECTS}

Five PR monkeys, 5 TE monkeys, and control monkeys N1-N10 were tested on the simple object discrimination and concurrent discrimination learning tasks. These 10 control monkeys included all of the monkeys with the same behavioral history tested in our laboratory to date.

BEHAVIORAL TESTING: SIMPLE

OBJECT DISCRIMINATION TASK

Monkeys were given four separate two-choice object discrimination tasks. Each discrimination task required monkeys to learn to discriminate a distinctive pair of objects, which could be learned by normal monkeys in a single day. The four dis- criminations consisted of a red versus a green peanut shell, a white versus a black rectangle, a pink versus a yellow plastic eggshell, and a piece of a real Oreo cookie versus a plastic cookie. For the first three pairs of objects, a raisin reward was concealed within the underside of the object. For the last pair, the monkey was allowed to take the Oreo cookie as his reward. Each discrimination pair was administered over two daily session of 20 trials each, with an ITI of 15-20 sec. Two days later, an additional session of 20 trials was administered. The position of the correct object varied pseudorandomly (over the left or right well; Gellerman 1933). An interval of 3 days elapsed between the administration of each pair. Performance was assessed as the percentage of correct responses for each day of testing.

\section{BEHAVIORAL TESTING: CONCURRENT DISCRIMINATION LEARNING TASK}

Monkeys were required to simultaneously learn eight pairs of objects. Presentation of the pairs was intermingled so that each pair was presented five times during the course of a single session (40 trials per day). The stimuli were chosen specifically so that there would be interference between pairs of objects, making this a much more difficult discrimination task than the simple object discrimination task. Stimulus features including color and shape were repeated between pairs of objects. Accordingly, successful performance on this task required the monkey to discriminate the stimuli along more than one dimension. During each trial, one pair of objects was presented over the lateral food wells, and the monkey had to displace the correct object to find a raisin reward. One object in each pair was always correct, and its left-right position varied according to a pseudorandom sequence (Gellerman 1933). Monkeys were tested until they reached a criterion of 39 correct responses in 40 consecutive trials during a single test session.

\section{Results}

\section{SIMPLE OBJECT DISCRIMINATION}

Figure 10A shows the performance of the three groups of monkeys on the object discrimination task across the 3 days of testing. Performance was averaged across the four object discriminations. Individual scores for monkeys in the PR, TE,

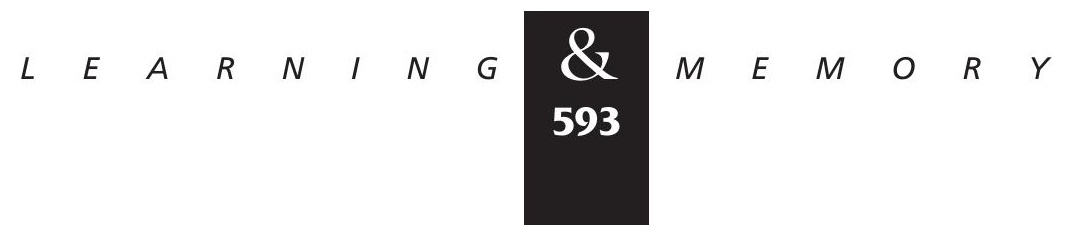




\section{Buffalo et al.}
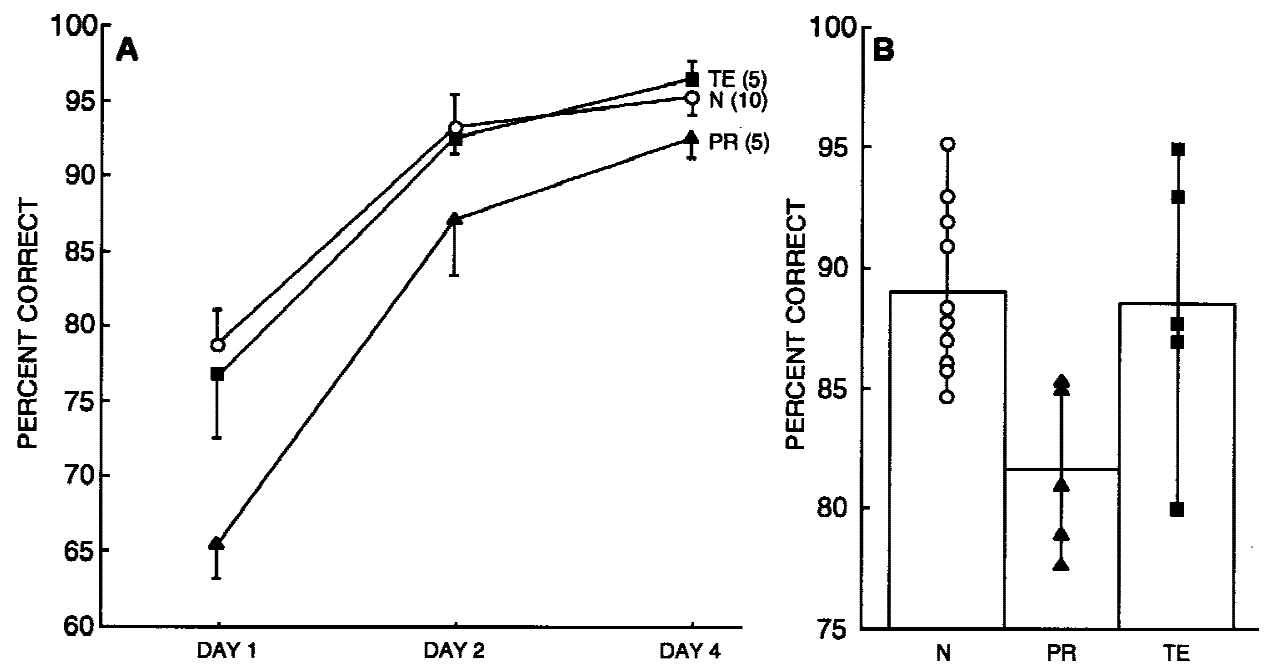

Figure 10: (A) Average daily performance on four simple object discrimination tasks by normal monkeys $(\mathrm{N}=10)$, monkeys with lesions of the perirhinal cortex $(P R=5)$, and monkeys with lesions of area TE $(T E=5)$. Bars represent the S.E.M. (B) Performance of the same monkeys averaged across all 3 days of testing. Symbols show the performance of individual monkeys (see also Table 1).

and $\mathrm{N}$ groups are shown in Table 1. A two-way ANOVA comparing the performance of the three groups of monkeys on the 3 days of the simple object discrimination task revealed a significant effect of group $[F(2,17)=5.68, P<0.05]$, a significant effect of day $[F(2,34)=79.14, P<0.05]$, and no significant group $\times$ day interaction $[F(2,34)=1.70$, $P>0.10]$. Separate comparisons based on each group's scores on each day of testing revealed that the PR group performed significantly worse than the $\mathrm{N}$ group and the TE group on day $1(\mathrm{PR}=65 \%$ correct, $\mathrm{TE}=77 \%$ correct, $\mathrm{N}=79 \%$ correct; $\mathrm{PR}$ vs. $\mathrm{N}, P<0.01$; PR vs. TE, $P<0.05)$. Additionally, the PR group performed significantly worse than the TE group on Day 4 ( $P R=92 \%$ correct, $T E=96 \%$ correct; $\mathrm{P}=0.05)$. There were no significant differences between the TE group and the $\mathrm{N}$ group on any of the testing days (all $P$ s $>0.10$ ).

Post-hoc analysis (Fisher's PLSD, two-tailed) revealed that when the 3 days of testing were considered together (Fig. 10B), the PR group was significantly impaired relative to both the $\mathrm{N}$ group and the TE group ( $\mathrm{PR}=82 \%$ correct, $\mathrm{TE}=89 \%$ correct, $\mathrm{N}=89 \%$ correct; $\mathrm{PR}$ vs. $\mathrm{N}, P<0.05$; $\mathrm{PR}$ vs. TE, $P<0.05$; TE vs. N, $P>0.10$ ).

Thus, compared with the $\mathrm{N}$ group, the $\mathrm{PR}$ group exhibited a deficit on the simple object discrimination task across the 3 testing days; this impairment was most evident on the first day of testing. In contrast, there were no differences between the performance of the TE group and the $\mathrm{N}$ group on this task. Importantly, because the PR group performed significantly worse than the TE group, this task demonstrated a dissociation between the effects of the PR and area TE lesions.

\section{CONCURRENT DISCRIMINATION LEARNING TASK}

Figure 11 shows the mean number of trials required by each group of monkeys to reach criterion level performance on the concurrent discrimination learning task. Individual scores for the $\mathrm{PR}, \mathrm{TE}$, and $\mathrm{N}$ groups are shown in Figure 11 and in Table 1. A one-way ANOVA comparing the performance of the three groups of monkeys revealed a significant effect of group $[F(2,17)=2.33$, $P<0.01]$. Post-hoc analysis (Fisher's PLSD, twotailed) revealed that the TE group was significantly impaired relative to the $\mathrm{N}$ group ( $\mathrm{TE}=933$ trials, $\mathrm{N}=417$ trials, $P<0.01)$. Additionally, the TE group was marginally impaired relative to the PR group ( $\mathrm{PR}=639, P=0.08)$. There was no significant difference between the $\mathrm{N}$ group and the $\mathrm{PR}$ group $(P>0.10)$.

In summary, this task provided an additional dissociation between the effects of PR and area TE lesions. The TE group was impaired on this task relative to the $\mathrm{N}$ group; in contrast, although two of the five monkeys did not perform well, the PR group as a whole was not significantly impaired. Moreover, the TE group was marginally impaired relative to the $\mathrm{PR}$ group.

$$
\begin{array}{llllllllllllllll} 
& E & A & R & N & I & N & G & \boldsymbol{Q} \\
\mathbf{5 9 4} & M & E & M & O & R & Y
\end{array}
$$




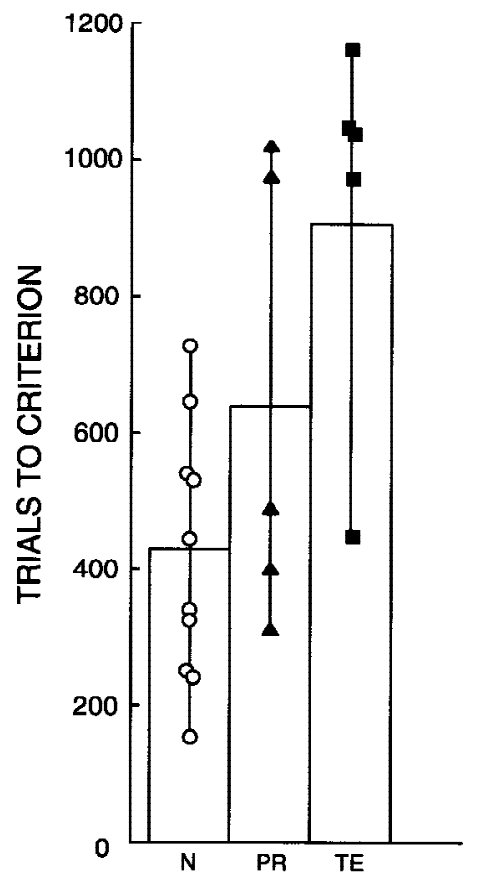

Figure 11: Performance on the concurrent discrimination learning task by normal monkeys $(N=10)$, monkeys with lesions of the perirhinal cortex $(P R=5)$, and monkeys with lesions of area TE $(\mathrm{TE}=5)$. Symbols show trials to criterion for individual monkeys.

\section{Discussion}

The findings from experiment 3 demonstrate additional dissociations between the effects of lesions of the perirhinal cortex and lesions of area TE. The PR group was impaired on the simple object discrimination task and unimpaired on the concurrent discrimination task. In contrast, the TE group was unimpaired on the simple object discrimination task and impaired on the concurrent discrimination task.

\section{General Discussion}

Table 2 summarizes the results from all three experiments. The PR and TE lesion groups differed with respect to their patterns of impairment (1st and 2nd columns, respectively). First, lesions of the perirhinal cortex impaired memory in both the visual and tactual modalities; lesions of area TE impaired memory only in the visual modality (experiment 1). Second, lesions of perirhinal cortex selectively impaired long-term visual recognition memory but spared short-term memory at delays of $1 \mathrm{sec}$; lesions of area TE impaired both short-term memory and long-term memory (experiment 2 ). Finally, lesions of perirhinal cortex impaired performance on the simple object discrimination task but spared performance on the concurrent discrimination task. Lesions of area TE produced the opposite pattern: Performance was spared on the simple object discrimination task but impaired on the concurrent discrimination task. Figure 12 highlights these four differences between the two lesion groups.

Why did TE lesions impair concurrent discrimination learning and short-term performance on the visual paired-comparison task but spare performance on the simple object discrimination task? We suggest that intact visual areas upstream from area TE, for example, areas TEO and V4, are suffi-

Table 2: Summary of behavioral findings

\begin{tabular}{lccc}
\hline & PR vs. N & TE vs. N & PR vs. TE \\
\hline Experiment 1 & & & \\
$\quad$ V-DNMS & + & + & \\
T-DNMS & + & - & + \\
Experiment 2 & & & \\
$\quad$ VPC (1-sec delay) & - & + & + \\
VPC (10 sec to 10 min) & + & + & \\
Experiment 3 & + & - & + \\
$\quad$ Simple object discrimination & + & + & $++^{a}$ \\
Concurrent discrimination & - & + & \\
\hline
\end{tabular}

Shading identifies instances where performance of the PR group differs from that of the TE group. (+) Impaired performance relative to normal monkeys, $P<0.05$. (-) Normal performance, $P>0.10$. (+) A significant difference between the PR and TE groups, $P<0.05$. ${ }^{\text {A }}$ marginally significant difference between the PR and TE groups, $P=0.08$. Abbreviations are as in Table 1.

$$
\begin{array}{llllllllllllllll} 
& E & A & R & N & I & N & G & \boldsymbol{Q} \\
\mathbf{5} 95 & M & E & M & O & R & Y
\end{array}
$$




\section{Buffalo et al.}

Figure 12: (A) Performance on the T-DNMS task (trials to criterion), the VPC task (1-sec delay), the simple object discrimination task (mean of 3 test days), and the concurrent discrimination learning task (trials to criterion) by monkeys with lesions of the perirhinal cortex (PR), monkeys with lesions of area TE (TE), and normal monkeys $(\mathrm{N})$. Asterisks $(*)$ denote a significant difference between the PR and TE groups and impaired performance relative to normal monkeys $(P<0.05)$. ( $\left.{ }^{\mathrm{a}}\right) \mathrm{A}$ marginally significant difference between the PR and TE groups; $P=0.08$. The numbers in parentheses indicate the number of monkeys in each group. Bars represent the S.E.M.
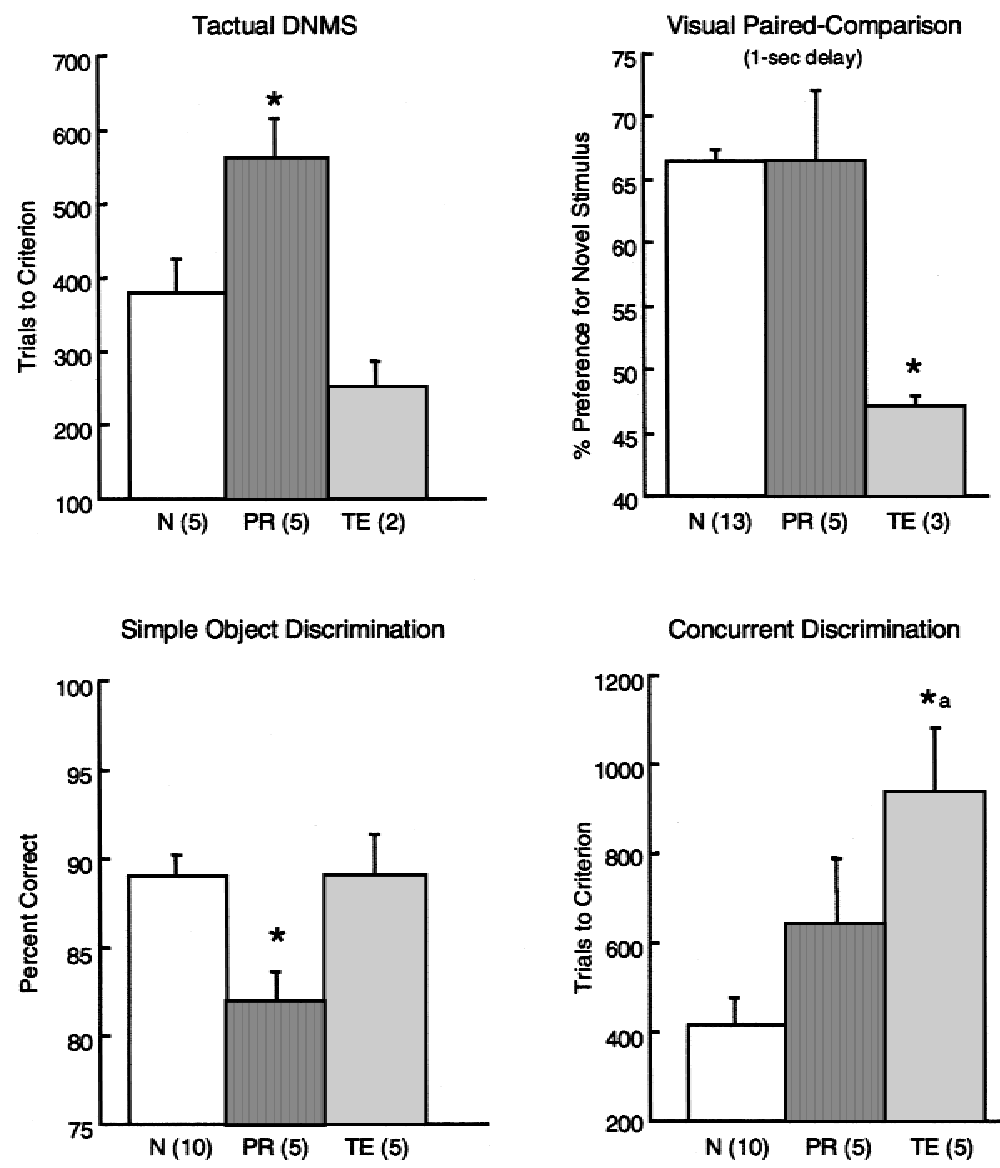

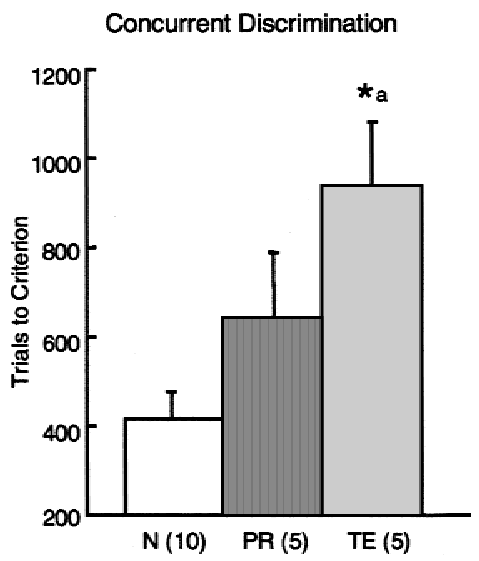

cient to process the relatively simple objects used in the simple object discrimination task. However, these upstream visual areas are not sufficient to process the more complex two-dimensional pattern stimuli used in the VPC task or the more complex, multiple objects used in the eight-pair concurrent discrimination task. Processing these stimuli requires area TE. Because areas TEO and V4 send direct projections to the perirhinal cortex ( $\mathrm{Su}-$ zuki and Amaral 1994a,b), visual information processed in these areas has access to the medial temporal lobe memory system, even in the presence of area TE lesions.

Why did perirhinal cortex lesions impair performance on the simple object discrimination task but spare short-term memory performance on the VPC task and spare concurrent discrimination learning? We suggest that short-term memory performance on the VPC task was spared because PR lesions cause primarily a memory problem and, like other medial temporal lobe lesions, do not impair performance at very short delays. We further suggest that PR lesions spared concurrent discrimi- nation learning because PR lesions impair declarative memory, and in the monkey the concurrent discrimination task does not provide a good test of this form of memory. This task has been considered previously to be a task of habit learning (Mishkin et al. 1984; Phillips et al. 1988; Buffalo et al. 1998b). In this task monkeys must learn how two stimuli are different and which differences are relevant to the task. This differentiation is accomplished gradually, day by day, over hundreds of trials, and learning improves progressively from chance to higher levels of performance. For discrimination learning of this kind, identifying which stimulus is the rewarded one is secondary to the gradual tuning in of the appropriate stimulus dimension.

In contrast, when only a single pair of objects needs to be learned and the objects are easily discriminable, as in the simple object discrimination task, learning which stimulus is correct becomes the major work of the task. By this formulation, an object discrimination that takes only 10-20 trials to learn (e.g., the simple object discrimination task)

$$
\begin{array}{lllllllllllllll}
L & E & A & R & N & I & N & G & \boldsymbol{Q} & M & E & M & O & R & Y \\
\mathbf{5 9 6} & & & & & & & & & & & & & &
\end{array}
$$


should be expected to be more sensitive to the effects of medial temporal lesions than a discrimination that takes 300-500 trials to learn (e.g., the concurrent discrimination task). An earlier review of the data from 46 two-choice discrimination tasks found that the tasks learned quickly were more sensitive to medial temporal lobe lesions than the tasks learned gradually (Squire and Zola-Morgan 1983; for a more recent discussion of this same idea, see Wise and Murray 1999). Finally, in other work, combined perirhinal and entorhinal cortex lesions in the monkey impaired visual discrimination learning when a single pair of stimuli was used and learning in normal animals occurred relatively quickly (Murray et al. 1998; Baxter et al. 1999).

The effects of area TE lesions were opposite to the effects of perirhinal cortex lesions. Lesions of area TE spared performance on the simple object discrimination task and impaired performance on tasks that involved more complex and potentially interfering stimuli (eight-pair concurrent discrimination learning). This pattern of findings is consistent with studies demonstrating that the effects of area TE lesions depend on the difficulty of the visual discrimination (Mishkin 1954; Mishkin and Pribram 1954; Mishkin and Hall 1955; Pribram and Mishkin 1955; Gross 1973).

The present interpretation that lesions of the perirhinal cortex impair declarative memory is different from earlier proposals that the perirhinal cortex is involved in the perceptual processing of visual stimuli (Eacott et al. 1994) or in the identification of individual objects (Buckley and Gaffan 1997, 1998a,b). According to these proposals, lesions of the perirhinal cortex do not selectively impair memory but instead produce a deficit that includes impaired visual perceptual processing or impaired object identification. The study by Eacott et al. (1994) assessed the performance of monkeys with combined lesions of the perirhinal and entorhinal cortex on five separate tasks of matching to sample that included simultaneous-matching and 0 -sec delay conditions. Delays of $5 \mathrm{sec}, 15 \mathrm{sec}$, and $30 \mathrm{sec}$ were also tested. Under simultaneous or 0-sec delay conditions, the matching-to-sample task purports to assess visual perceptual ability rather than memory. When performance on the simultaneous-matching-to-sample and the 0-sec delay conditions were combined across two of the five tasks (tasks A and D in Eacott et al. 1994), the operated monkeys were impaired.

However, this study does not provide strong evidence for an impairment in visual perceptual processing in the operated monkeys. First, the simultaneous-matching and 0-sec delay conditions in the matching-to-sample task do not provide an unambiguous assessment of the capacity for visual perceptual processing. Specifically, the task requires not only visual perceptual processing but also the retention and sustained application of the matching rule. Diminished performance on the simultaneous and 0 -sec delay conditions could therefore reflect either a visual perceptual impairment or a difficulty retaining and implementing the matching rule. Second, it is unclear whether the scores of the operated animals reflect significant impairment. Performance measures for the simultaneous-matching and 0-sec delay conditions were obtained on all five tasks. By inspecting the relavant figures, we estimate that the average difference between the scores of the operated and normal groups was only $4 \%$ for the simultaneousmatching condition (task $\mathrm{A}=5 \%$, task $\mathrm{C}=3 \%$, task $\mathrm{A} 1=1 \%$, task $\mathrm{D}=11 \%$, task $\mathrm{E}=1 \%)$ and $6 \%$ for the 0 -sec delay condition (task $\mathrm{A}=5 \%$, task $\mathrm{C}=11 \%$, task $\mathrm{A} 1=8 \%$, task $\mathrm{D}=7 \%$, task $\mathrm{E}=0 \%$. No statistical data were reported, except for the analysis that combined only tasks $\mathrm{A}$ and $\mathrm{D}$.

Buckley and Gaffan suggested that perirhinal cortex lesions impair performance only when the monkey's ability to identify individual objects is sufficiently taxed, either by increasing the number of stimuli to be discriminated (Buckley and Gaffan 1997), by presenting the object from an unusual view (Buckley and Gaffan 1998a,b), or by presenting the object in an unfamiliar context (Buckley and Gaffan 1998a). However, a clear interpretation of the findings from the studies by Buckley and Gaffan is difficult because all three monkeys with perirhinal cortex lesions that participated in these studies sustained inadvertent damage to area TE. Based on the available neurohistological information from the reports by Buckley and Gaffan, the amount of TE damage in the three animals with perirhinal cortex lesions appears sufficient to account for the observed impairments (see Buffalo et al. 1998b). Based on the scale used by Buffalo et al. (1998b), the amount of inadvertent area TE damage in these animals was rated as follows: PRh1 $=1.4, \mathrm{PRh} 2=1.3, \mathrm{PRh} 3=1.17$. This amount of damage to area TE is sufficient to produce impaired performance on the concurrent discrimination learning task (see Buffalo et al. 1998b; Fig. 6A).

In summary, the present findings demonstrate the distinct functions of the perirhinal cortex and area TE. The deficits following lesions of area TE

$$
\begin{array}{lllllllllllllll}
L & E & A & R & N & I & N & G & \mathbf{Q} & M & E & M & O & R & Y \\
\mathbf{5 9 7} & & & & & & &
\end{array}
$$




\section{Buffalo et al.}

are consistent with an impairment of visual perception. Lesions of area TE produced a unimodal visual impairment in visual recognition memory (experiment 1), an impairment in visual recognition even at very short delays (experiment 2), and an impairment in relatively complex visual discrimination learning together with sparing of simple visual object discrimination learning (experiment 3). In contrast, the impairment following perirhinal lesions is best described as a memory impairment. Lesions of perirhinal cortex produced a multimodal impairment in recognition memory (experiment 1), spared short-term memory and impaired long-term memory (experiments 1 and 2), and impaired simple object discrimination learning while sparing more complex visual discrimination learning (experiment 3).

\section{Acknowledgments}

This research was supported by the Medical Research Service of the Department of Veterans Affairs, National Institutes of Health $(\mathrm{NIH})$ grants $\mathrm{MH} 24600$ and 2T32AG00216 (E.A.B.), and the McDonnell-Pew Center for Cognitive Neuroscience (E.A.B., S.J.R., E.T.). We thank David Amaral for preparation of the perirhinal cortex lesions and Lisa Stefanacci for assistance with histological analysis. We also thank Cecelia Manzanares, Jeff Manzanares, Scott Hanson, Lawrence Truong, Alisha West, and Michelle Hu for excellent technical assistance.

The publication costs of this article were defrayed in part by payment of page charges. This article must therefore be hereby marked "advertisement" in accordance with 18 USC section 1734 solely to indicate this fact.

\section{References}

Bachevalier, J., M. Brickson, and C. Hagger. 1993. Limbic-dependent recognition memory in monkeys develops early in infancy. NeuroReport 4: 77-80.

Baxter, M.G., W.S. Hadfield, and E.A. Murray. 1999. Rhinal cortex lesions produce mild deficits in visual discrimination learning for auditory secondary reinforcer in rhesus monkeys. Behav. Neurosci. 113: 243-252.

Brodmann, K. 1909. Vergleichende Lokalisationslehre der Grosshirnrinde in ihren Prinzipien dargestellt auf Grund des zellenbaues. Barth, Leipzig, Germany.

Buckley, M.J. and D. Gaffan. 1997. Impairment of visual object-discrimination learning after perirhinal cortex ablation. Behav. Neurosci. 111: 467-475.

1998a. Learning and transfer of object-reward associations and the role of the perirhinal cortex. Behav. Neurosci. 112: 15-23.

1998b. Perirhinal cortex ablation impairs visual object identification. J. Neurosci. 18: 2268-2275.
Buckley, M.J., D. Gaffan, and E.A. Murray. 1997. Functional double dissociation between two inferior temporal cortical areas: Perirhinal cortex versus middle temporal gyrus. J. Neurophysiol. 77: 587-597.

Buffalo, E.A., P. Reber, and L.R. Squire. 1998a. The human perirhinal cortex and visual recognition memory. Hippocampus 8: 330-339.

Buffalo, E.A., L. Stefanacci, L.R. Squire, and S.M. Zola. 1998b. A reexamination of the concurrent discrimination learning task: The importance of inferotemporal cortex, area TE. Behav. Neurosci. 112: 3-14.

Dean, P. 1976. Effects of inferotemporal lesions on the behavior of monkeys. Psychol. Bull. 83: 41-71.

Eacott, M.J., D. Gaffan, and E.A. Murray. 1994. Preserved recognition memory for small sets, and impaired stimulus identification for large sets, following rhinal cortex ablations in monkeys. Eur. J. Neurosci. 6: 1466-1478.

Ennaceur, A. and J.P. Aggleton. 1997. The effects of neurotoxic lesions of the perirhinal cortex combined to fornix transection on object recognition memory in the rat. Behav. Brain Res. 88: 181-193.

Ennaceur, A., N. Neave, and J.P. Aggleton. 1996. Neurotoxic lesions of the perirhinal cortex do not mimic the behavioral effects of formix transection in the rat. Behav. Brain Res. 80: 9-25.

Fuster, J.M., R.H. Bauer, and J.P. Jervey. 1981. Effects of cooling inferotemporal cortex on performance of visual memory tasks. Exp. Neurol. 71: 398-409.

Gellerman, L.W. 1933. Chance orders of alternating stimuli in visual discrimination experiments. J. Genet. Psychol. 42: 207-208.

Goulet, S. and E.A. Murray. 1996. Neural substrate of intramodal and crossmodal memory in rhesus monkeys. Int. J. Psychol. 31: 307.

Gross, C.G. 1973. Visual functions of inferotemporal cortex. In Handbook of sensory physiology, Vol. VII/3B (ed. R. Jung), pp. 451-482. Springer Verlag, Berlin, Germany.

Harlow, H. and J.A. Bromer. 1938. A test-apparatus for monkeys. Psychol. Rev. 19: 434-438.

Horel, J.A., D.E. Pytko-Joiner, M.L. Voytko, and K. Salsbury. 1987. The performance of visual tasks while segments of the inferotemporal cortex are suppressed by cold. Behav. Brain Res. 23: 29-42.

Iwai, E. and M. Mishkin. 1968. Two visual foci in the temporal lobe of monkeys. In Neurophysiological basis of learning and behavior (ed. N. Yoshii and N.A. Buchwald), pp. 1-11. Osaka University Press, Osaka, Japan.

Malkova, L., M. Mishkin, and J. Bachevalier. 1995. Long-term effects of selective neonatal temporal lobe lesions on learning and memory in monkeys. Behav. Neurosci. 109: 212-226.

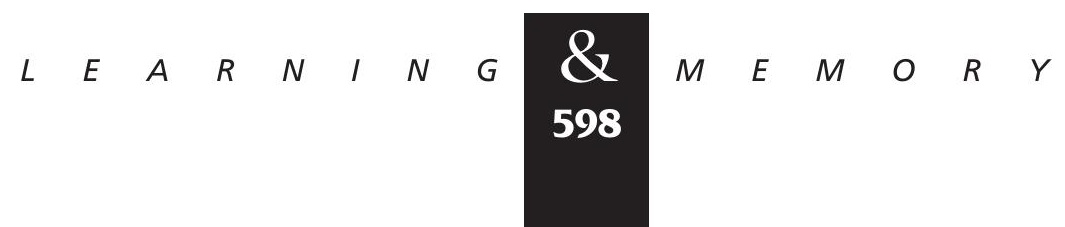


Meunier, M.J., J. Bachevalier, M. Mishkin and E.A. Murray. 1993. Effects on visual recognition of combined and separate ablations of the entorhinal and perirhinal cortex in rhesus monkeys.J. Neurosci.13: 5418-5432.

Milner, B. 1972. Disorders of learning and memory after temporal lobe lesions in man. In Clinical neurosurgery 19: 421-446.

Mishkin, M. 1954. Visual discrimination performance following partial ablations of the temporal lobe: II.Ventral surface vs. hippocampus. J. Comp. Physiol. Psychol. 47: 187-193.

1982. A memory system in the monkey. Phil. Trans. R. Soc. Lond. B. 298: 85-92.

Mishkin, M., and K.H. Pribram. 1954. Visual discrimination performance following partial ablations of the temporal lobe: I.Ventral vs. lateral. J. Comp. Physiol. Psychol. 47: 14-20.

Mishkin, M. and M. Hall. 1955. Discrimination along a size continuum following ablation of the inferior temporal convexity in monkeys. J. Comp. Physiol. Psychol.

48: 97-101.

Mishkin, M. and E.A. Murray. 1994. Stimulus recognition. Curr. Opin. Neurobiol. 4: 200-206.

Mishkin, M., B. Malamut, and J. Bachevalier. 1984. Memories and habits: Two neural systems. In Neurobiology of human learning and memory (ed. G. Lynch, J.L. McGaugh, and N.M. Weinberger), pp. 65-77. Guilford Press, New York, N.Y.

Miyashita, Y. 1993. Inferior temporal cortex: Where perception meets memory. Annu. Rev. Neurosci. 16: $245-264$.

Mumby, D.G. and J.P.J. Pinel. 1994. Rhinal cortex lesions and object recognition in rats. Behav. Neurosci. 108: 11-18.

Murray, E.A. and M. Mishkin. 1984. Severe tactual as well as visual memory deficits follow combined removal of the amygdala and hippocampus in monkey. J. Neurosci. 4: 2565-2580.

Murray, E.A., M.G. Baxter, and D. Gaffan. 1998. Monkeys with rhinal cortex damage or neurotoxic hippocampal lesions are impaired on spatial scene learning and object reversals. Behav. Neurosci. 112: 1291-1303.

Phillips, R.R., B.L. Malamut, J. Bachevalier, and M. Mishkin. 1988. Dissociation of the effects of inferior temporal and limbic lesions on object discrimination learning with 24-hr intertrial intervals. Behav. Brain Res. 27: 99-107.

Pribram, K.H. and M. Mishkin. 1955. Simultaneous and successive visual discrimination by monkeys with inferotemporal lesions. J. Comp. Physiol. Psychol. 48: 198-202.
Saint-Cyr, J.A., L.G. Ungerleider and R. Desimone. 1990. Organization of visual inputs to the striatum and subsequent outputs to the pallido-nigral complex in the monkey. J. Comp. Neurol. 298: 129-156.

Squire, L.R. and S. Zola-Morgan. 1983. The neurology of memory: The case for correspondence between the findings for human and nonhuman primate. In The physiological basis of memory (ed. J.A. Deutsch), pp. 199-268. Academic Press, New York, NY.

Squire, L.R. and S.M. Zola. 1997. Amnesia, memory and brain systems. Phil. Trans. R. Soc. Lond. B. 352: 1663-1673.

Suzuki, W.A. and D.G. Amaral. 1990. Cortical inputs to the CA1 field of the monkey hippocampus originate from the perirhinal and parahippocampal cortex but not from area TE. Neurosci. Lett. 115: 43-48.

Suzuki, W.A. and D.G. Amaral . 1994a. The perirhinal and parahippocampal cortices of the macaque monkey: Cortical afferents. J. Comp. Neurol. 350: 497-533.

1994b. Topographic organization of the reciprocal connections between the monkey entorhinal cortex and the perirhinal and parahippocampal cortices. J. Neurosci.

14: $1856-1877$.

Suzuki, W.A., S. Zola-Morgan, L.R. Squire, and D.G. Amaral. 1993. Lesions of the perirhinal and parahippocampal cortices in the monkey produce long-lasting memory impairment in the visual and tactual modalities. J. Neurosci.

13: $2430-2451$.

Szabo, J. and W.M. Cowan. 1984. A stereotaxic atlas of the brain of the cynomolgus monkey (Macaca fascicularis). J. Comp. Neurol. 222: 265-300.

Ungerleider, L.G. and M. Mishkin. 1982. Two cortical visual systems. In The analysis of visual behavior (ed. D.J. Ingle, R.J.W. Mansfield, and M.A. Goodale), pp. 549-586. MIT Press, Cambridge, MA.

Van Hoesen, G.W., E.H. Yeterian, and R. Lavizzo-Mourey. 1981. Widespread corticostriate projections from temporal cortex of the rhesus monkey. J. Comp. Neurol. 199: 205-219.

Webster, M.J., J. Bachevalier, and L.G. Ungerleider. 1994. Connections of inferior temporal areas TEO and TE with parietal and frontal cortex in Macaque monkeys. Cereb. Cortex 5: 479-483.

Wise, S.P. and E.A. Murray. 1999. Role of the hippocampal system in conditional motor learning: Mapping antecedents to action. Hippocampus 9: 101-117.

Received August 30, 1999; accepted in revised form October 21, 1999.

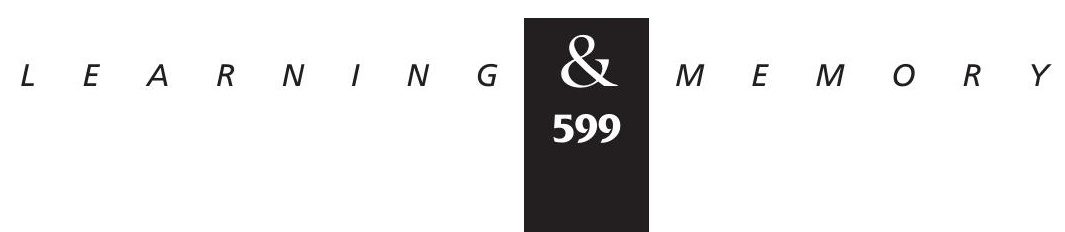




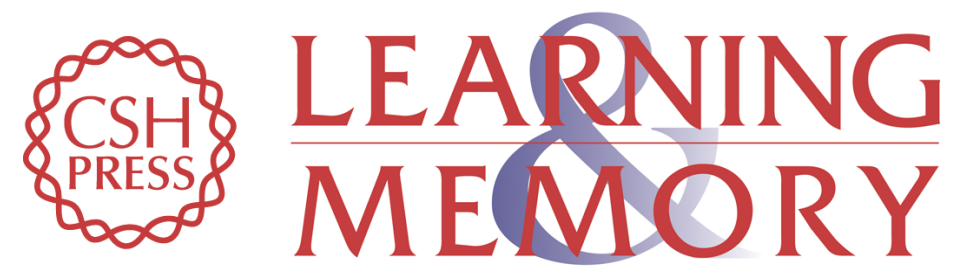

\section{Dissociation Between the Effects of Damage to Perirhinal Cortex and Area TE}

Elizabeth A. Buffalo, Seth J. Ramus, Robert E. Clark, et al.

Learn. Mem. 1999, 6:

Access the most recent version at doi:10.1101//m.6.6.572

References This article cites 39 articles, 5 of which can be accessed free at: http://learnmem.cshlp.org/content/6/6/572.full.html\#ref-list-1

License

Email Alerting Receive free email alerts when new articles cite this article - sign up in the box at the Service top right corner of the article or click here. 